\title{
A New Result Concerning the Solvability of a Class of General Systems of Variational Equations with Nonmonotone Operators: Applications to Dirichlet and Neumann Nonlinear Problems
}

\author{
Luisa Toscano ${ }^{1}$ and Speranza Toscano $^{2}$ \\ ${ }^{1}$ Department of Mathematics and Applications "R. Caccioppoli", University of Naples "Federico II", Via Cintia, \\ Monte S. Angelo, I-80126 Naples, Italy \\ ${ }^{2}$ University of Naples, Via Roma 29, 81031 Aversa, Italy
}

Correspondence should be addressed to Luisa Toscano; luisa.toscano2@unina.it

Received 30 July 2015; Accepted 28 September 2015

Academic Editor: Jianshe Yu

Copyright ( 92016 L. Toscano and S. Toscano. This is an open access article distributed under the Creative Commons Attribution License, which permits unrestricted use, distribution, and reproduction in any medium, provided the original work is properly cited.

A new result of solvability for a wide class of systems of variational equations depending on parameters and governed by nonmonotone operators is found in a Banach real and reflexive space with applications to Dirichlet and Neumann problems related to nonlinear elliptic systems.

\section{Introduction}

Many researchers (e.g., [1-9]) have devoted (and are still devoting) their studies to the solvability and the investigation of multiple and positive solutions of nonlinear elliptic problems.

A class of general systems of variational equations has been studied in [10]. The Dirichlet and Neumann problems investigated in [11] belong to this class.

In this paper, we prove a further existence theorem related to the problem of [10] in the homogeneous case, by using the Lagrange multipliers and the "algebraic" approach which is based on the fibering method [12]. This theorem and the ones of [10] include the results of [8] and some results of [13-15]. Now, let us recall the problem studied in [10].

Let $\left(W_{1},\|\cdot\|_{1}\right), \ldots,\left(W_{n},\|\cdot\|_{n}\right)(n \geq 1)$ be Banach reflexive and real spaces. Let $W=X_{\ell=1}^{n} W_{\ell}$ with $\|v\|=\sum_{\ell=1}^{n}\left\|v_{\ell}\right\|_{\ell} \forall v=$ $\left(v_{1}, \ldots, v_{n}\right) \in W$. Let $\langle\cdot, \cdot\rangle_{\ell}[$ resp., $\langle\langle\cdot, \cdot\rangle]$ be the duality between $W_{\ell}^{*}$ dual space of $W_{\ell}$ [resp., $W^{*}$ dual space of $W$ ] and $W_{\ell}$ [resp., $\left.W\right]$. Let us denote by " $\partial$ " Fréchet differential operator and by " $\partial_{u_{\rho}}$ " Fréchet differential operator with respect to $u_{\ell}$. Let $A \neq \equiv 0$ and $D_{j} \neq \equiv(j=1, \ldots, m ; m \geq 1)$ be real functionals defined in $W$ and let $B_{\ell}$ and $\widehat{B}_{\ell}(\ell=1, \ldots, n)$ be real functionals defined in $W_{\ell}$ satisfying the following conditions:

$\left(i_{11}\right) A$ is lower weakly semicontinuous in $W$ and $C^{1}(W \backslash$ $\{0\}), B_{\ell}$ and $\widehat{B}_{\ell}$ are weakly continuous in $W_{\ell}$ and $C^{1}\left(W_{\ell}\right), \exists p>1: A(t v)=t^{p} A(v) \forall t \geq 0$ and $\forall v \in W$, $B_{\ell}\left(t v_{\ell}\right)=t^{p} B_{\ell}\left(v_{\ell}\right)$ and $\widehat{B}_{\ell}\left(t v_{\ell}\right)=t^{p} \widehat{B}_{\ell}\left(v_{\ell}\right) \forall t \geq 0$ and $\forall v_{\ell} \in W_{\ell}$.

$\left(i_{12}\right) D_{j}$ is weakly continuous in $W$ and $C^{1}(W \backslash\{0\}), \exists q_{j}>$ $1: D_{j}(t v)=t^{q_{j}} D_{j}(v) \forall t \geq 0$, and $\forall v \in W, 1<q_{1}<$ $\cdots<q_{m}$ if $m>1$. 
Let us set the following:

$$
\begin{aligned}
& H_{\lambda \mu}(v)=A(v)-\sum_{\ell=1}^{n}\left[\lambda_{\ell} B_{\ell}\left(v_{\ell}\right)+\mu_{\ell} \widehat{B}_{\ell}\left(v_{\ell}\right)\right] \\
& E(v)=H_{\lambda \mu}(v)-\sum_{j=1}^{m} D_{j}(v) \\
& \forall v=\left(v_{1}, \ldots, v_{n}\right) \in W \text {, where } \lambda=\left(\lambda_{1}, \ldots, \lambda_{n}\right), \mu=\left(\mu_{1}, \ldots, \mu_{n}\right) \in R^{n} \text {; } \\
& S_{\lambda \mu}=\left\{v \in W: H_{\lambda \mu}(v)=1\right\} \text {, } \\
& V_{\lambda \mu}^{-}=\left\{v \in W: H_{\lambda \mu}(v)<0\right\}, \\
& S\left(D_{1}\right)=\left\{v \in W: D_{1}(v)=-1\right\} \text {, } \\
& V^{+}\left(D_{1}, \ldots, D_{m_{1}}\right)=\left\{v \in W: \sum_{j=1}^{m_{1}} D_{j}(v)>0\right\} \quad \text { as } m_{1}=1, \ldots, m \text {. }
\end{aligned}
$$

Let us consider the following problem.

Problem $(P)$. Find $u=\left(u_{1}, \ldots, u_{n}\right) \in W \backslash\{0\}$ such that

$$
\begin{array}{r}
\left\langle\partial_{u_{i}} A(u), v_{i}\right\rangle_{i}=\lambda_{i}\left\langle\partial B_{i}\left(u_{i}\right), v_{i}\right\rangle_{i}+\mu_{i}\left\langle\partial \widehat{B}_{i}\left(u_{i}\right), v_{i}\right\rangle_{i} \\
+\sum_{j=1}^{m}\left\langle\partial_{u_{i}} D_{j}(u), v_{i}\right\rangle_{i} \\
\forall i \in\{1, \ldots, n\}, \forall v_{i} \in W_{i} .
\end{array}
$$

In Section 2, we present new cases $\left(\left(c_{1}\right)-\left(c_{4}\right)\right)$ in which Problem (P) is solvable. In these cases, we introduce one of the following hypotheses:

$$
\begin{aligned}
& \left(i_{13}\right) \exists c(\lambda, \mu)>0:\|v\|^{p} \leq c(\lambda, \mu) H_{\lambda \mu}(v) \forall v \in W \\
& \left(i_{14}\right) \exists c(\lambda, \mu)>0:\|v\|^{p} \leq c(\lambda, \mu) H_{\lambda \mu}(v) \forall v \in V^{+}\left(D_{1}\right) \\
& \quad\left(\text { if } V^{+}\left(D_{1}\right) \neq \varnothing\right) .
\end{aligned}
$$

Theorem 1 assures the existence of at least one solution. It is possible to get the existence of a second solution from a result of [10, Theorem 2.2]. This result is based in particular on the following assumption:

$$
\left(i_{15}\right) V_{\lambda \mu}^{-} \cap S\left(D_{1}\right) \text { is not empty and bounded in } W \text {. }
$$

The applications to Dirichlet problems in Section 3 [resp., Neumann problems in Section 4] (whose variational form is included in Problem (P)), for the sake of brevity, deal with the first case of solvability, since in this case we can use at the same time Theorems 1 and 4 . When $n>1$, thanks to Propositions 2 and 5, we have got sufficient conditions so that the components of the found solutions are not identically equal to zero.

\section{Solvability of Problem (P)}

Let us consider the following cases:

$\left(c_{1}\right) m>1, q_{1}<p, V^{+}\left(D_{1}\right) \neq \varnothing, D_{j} \leq 0 \forall j \in\{2, \ldots, m\}$, and $\left(i_{14}\right)$ holds.

$\left(c_{2}\right) m>1, \exists m_{1} \in\{2, \ldots, m\}: q_{m_{1}}<p, D_{j} \geq 0 \forall j \epsilon$ $\left\{1, \ldots, m_{1}\right\}, D_{j} \leq 0 \forall j \in\left\{m_{1}+1, \ldots, m\right\}$ if $m_{1}<m$, and $\left(i_{13}\right)$ holds.

$\left(c_{3}\right) m>1, q_{m}<p, D_{j} \geq 0 \forall j \in\{1, \ldots, m-1\}$, $\sum_{j=1}^{m-1} D_{j}(v)>0 \forall v \in W \backslash\{0\}, D_{m}$ changes sign, and $\left(i_{13}\right)$ holds.

$\left(c_{4}\right) m>1, p<q_{1}, D_{1}$ changes sign, $D_{j} \geq 0 \forall j \epsilon$ $\{2, \ldots, m\}, \sum_{j=2}^{m} D_{j}(v)>0 \forall v \in W \backslash\{0\}$, and $\left(i_{13}\right)$ holds.

Let us introduce the open set $\mathscr{A}$ of the space $W$ :

$$
\begin{aligned}
& \mathscr{A}=V^{+}\left(D_{1}\right) \quad \text { in }\left(c_{1}\right), \\
& \mathscr{A}=V^{+}\left(D_{1}, \ldots, D_{m_{1}}\right) \quad \text { in }\left(c_{2}\right), \\
& \mathscr{A}=W \backslash\{0\} \quad \text { in }\left(c_{3}\right),\left(c_{4}\right),
\end{aligned}
$$

and let us set

$$
\begin{aligned}
& \widetilde{E}(t, v)=E(t v)=t^{p} H_{\lambda \mu}(v)-\sum_{j=1}^{m} t^{q_{j}} D_{j}(v), \\
& \psi(t, v)=\frac{\partial \widetilde{E}}{\partial t}(t, v),
\end{aligned}
$$

$\forall t \geq 0, \forall v \in \mathscr{A}$.

It is easy to control that, for each $v \in \mathscr{A}$, the equation

$$
\psi(t, v)=0
$$


has only one positive root $t(v)$ and it results in

$$
\begin{aligned}
& \frac{\partial \psi}{\partial t}(t(v), v)>0, \\
& \widetilde{E}(t(v), v)=\min _{t \geq 0} \widetilde{E}(t, v)<0 \\
& \quad \text { in }\left(c_{1}\right)-\left(c_{3}\right), \\
& \frac{\partial \psi}{\partial t}(t(v), v)<0, \\
& \widetilde{E}(t(v), v)=\max _{t \geq 0} \widetilde{E}(t, v)>0
\end{aligned}
$$

$$
\text { in }\left(c_{4}\right) \text {. }
$$

The implicit function theory assures the functional $t(v)$ belongs to $C^{1}(\mathscr{A})$. Then, the functional $\widetilde{\widetilde{E}}(v)=\widetilde{E}(t(v), v)$ belongs to $C^{1}(\mathscr{A})$ and it results in

$$
\begin{aligned}
& \ll \partial \widetilde{\widetilde{E}}(u), v 》) \\
& =\left[p(t(u))^{p-1} H_{\lambda \mu}(u)-\sum_{j=1}^{m} q_{j}(t(u))^{q_{j}-1} D_{j}(u)\right] \\
& \cdot\left\langle\partial t(u), v 》+(t(u))^{p} 《 \partial H_{\lambda \mu}(u), v 》\right) \\
& -\sum_{j=1}^{m}(t(u))^{q_{j}}\left\langle\left\langle\partial D_{j}(u), v 》=(t(u))^{p}\right.\right. \\
& \cdot\left\langle\partial H_{\lambda \mu}(u), v 》-\sum_{j=1}^{m}(t(u))^{q_{j}}\left\langle\partial D_{j}(u), v 》\right\rangle\right.
\end{aligned}
$$

$\forall u \in \mathscr{A}, \forall v \in W$

It is important to remark that (9) can also be written as follows:

$$
\begin{aligned}
& \langle\partial \widetilde{\widetilde{E}}(u), v\rangle=(t(u)) \\
& \left.\quad\left[\left\langle\partial H_{\lambda \mu}(t(u) u), v\right\rangle-\sum_{j=1}^{m}\left\langle\partial D_{j}(t(u) u), v\right\rangle\right]\right]
\end{aligned}
$$

$\forall u \in \mathscr{A}, \forall v \in W$

Theorem 1. Under assumptions $\left(i_{11}\right),\left(i_{12}\right)$, in cases $\left(c_{1}\right)-\left(c_{4}\right)$, one has

$$
\begin{aligned}
\exists v^{0} & =\left(v_{1}^{0}, \ldots, v_{n}^{0}\right) \in S_{\lambda \mu} \cap \mathscr{A}: \\
\widetilde{\widetilde{E}}\left(v^{0}\right) & =\inf \left\{\widetilde{\widetilde{E}}(v): v \in S_{\lambda \mu} \cap \mathscr{A}\right\}, \\
u^{0} & =t\left(v^{0}\right) v^{0} \text { is solution of Problem }(P) .
\end{aligned}
$$

Proof. Let us set

$$
\begin{aligned}
\underline{e} & =\inf \left\{\tilde{\widetilde{E}}(v): v \in S_{\lambda \mu} \cap \mathscr{A}\right\}, \\
\widetilde{E}_{1}(t, v) & =t^{p}-\sum_{j=1}^{m} t^{q_{j}} D_{j}(v), \\
\psi_{1}(t, v) & =\frac{\partial \widetilde{E}_{1}}{\partial t}(t, v) \\
\widetilde{\widetilde{E}}_{1}(v) & =\widetilde{E}_{1}\left(t_{1}(v), v\right) \quad \forall v \in \mathscr{A}, \quad \forall t \geq 0, \forall v \in \mathscr{A},
\end{aligned}
$$

where $t_{1}(v)$ is the positive root of equation $\psi_{1}(t, v)=0$. Pointing out that $\widetilde{\widetilde{E}}_{1} \in C^{1}(\mathscr{A})$ and

$$
\widetilde{\widetilde{E}}(v)=\widetilde{\widetilde{E}}_{1}(v) \quad \forall v \in S_{\lambda \mu} \cap \mathscr{A},
$$

let us prove that

$$
\begin{array}{r}
\exists v^{0} \in \mathscr{A}: \\
\widetilde{\widetilde{E}}_{1}\left(v^{0}\right)=\underline{e} .
\end{array}
$$

Let $\left\{v^{k}\right\} \subseteq S_{\lambda \mu} \cap \mathscr{A}$ such that $\widetilde{\widetilde{E}}_{1}\left(v^{k}\right) \rightarrow \underline{e}$; that is,

$$
\left(t_{1}\left(v^{k}\right)\right)^{p}-\sum_{j=1}^{m}\left(t_{1}\left(v^{k}\right)\right)^{q_{j}} D_{j}\left(v^{k}\right) \longrightarrow \underline{e} .
$$

We note that

$$
p\left(t_{1}\left(v^{k}\right)\right)^{p-1}-\sum_{j=1}^{m} q_{j}\left(t_{1}\left(v^{k}\right)\right)^{q_{j}-1} D_{j}\left(v^{k}\right)=0
$$

moreover,

$$
\left\|v^{k}\right\|^{p} \leq c(\lambda, \mu)
$$

since $\left(i_{14}\right)$ holds in $\left(c_{1}\right)$ and $\left(i_{13}\right)$ holds in $\left(c_{2}\right)-\left(c_{4}\right)$.

Inequality (18) implies that $v^{0} \in W$ exists such that (within a subsequence) $v^{k} \rightarrow v^{0}$ weakly in $W$. Then,

$$
\begin{aligned}
0 & \leq H_{\lambda \mu}\left(v^{0}\right) \leq 1, \\
D_{j}\left(v^{k}\right) & \longrightarrow D_{j}\left(v^{0}\right) \quad \text { as } j=1, \ldots, m .
\end{aligned}
$$

Let us verify that

$$
\sup _{1}\left(v^{k}\right)<+\infty
$$

In $\left(c_{1}\right)-\left(c_{3}\right)$, let $\delta>0$ such that $\left|D_{j}(v)\right| \leq \delta \forall v \in S_{\lambda \mu} \cap \mathscr{A}$ and $\forall j \in\{1, \ldots, m\}$. We set $\forall t \geq 0, \Phi(t)=p t^{p-1}-\delta q_{1} t^{q_{1}-1}$, in $\left(c_{1}\right)$, and $\Phi(t)=p t^{p-1}-\delta \sum_{j=1}^{m_{1}} q_{j} t^{q_{j}-1}$ in $\left(c_{2}\right), \Phi(t)=p t^{p-1}-$ $\delta \sum_{j=1}^{m} q_{j} t^{q_{j}-1}$ in $\left(c_{3}\right)$.

Let us denote by $t_{0}$ the positive root of equation $\Phi(t)=0$. Since $\psi_{1}(t, v) \geq \Phi(t) \forall t \geq 0$ and $\forall v \in S_{\lambda_{\mu}} \cap \mathscr{A}$, we have $t_{1}\left(v^{k}\right) \leq t_{0} \forall k \in N$. 
In $\left(c_{4}\right)$, we note that from $(17)\left(t_{1}\left(v^{k}\right)\right)^{q_{1}} D_{1}\left(v^{k}\right)=$ $\left(p / q_{1}\right)\left(t_{1}\left(v^{k}\right)\right)^{p}-\sum_{j=2}^{m}\left(q_{j} / q_{1}\right)\left(t_{1}\left(v^{k}\right)\right)^{q_{j}} D_{j}\left(v^{k}\right)$; then,

$$
\begin{aligned}
& \left(t_{1}\left(v^{k}\right)\right)^{p}-\sum_{j=1}^{m}\left(t_{1}\left(v^{k}\right)\right)^{q_{j}} D_{j}\left(v^{k}\right) \\
& =\left(1-\frac{p}{q_{1}}\right)\left(t_{1}\left(v^{k}\right)\right)^{p} \\
& \quad+\sum_{j=2}^{m}\left(\frac{q_{j}}{q_{1}}-1\right)\left(t_{1}\left(v^{k}\right)\right)^{q_{j}} D_{j}\left(v^{k}\right) \\
& \geq\left(1-\frac{p}{q_{1}}\right)\left(t_{1}\left(v^{k}\right)\right)^{p}
\end{aligned}
$$

from which (21) follows taking into account (16).

Relation (21) assures that $\omega \in[0,+\infty[$ exists such that (within a subsequence) $t_{1}\left(v^{k}\right) \rightarrow \omega$.

Consequently, from (16), (17), and (20), we obtain

$$
\begin{array}{r}
\omega^{p}-\sum_{j=1}^{m} \omega^{q_{j}} D_{j}\left(v^{0}\right)=\underline{e}, \\
p \omega^{p-1}-\sum_{j=1}^{m} q_{j} \omega^{q_{j}-1} D_{j}\left(v^{0}\right)=0 .
\end{array}
$$

Let us add that

$$
\begin{gathered}
\omega>0, \\
v^{0} \in \mathscr{A} .
\end{gathered}
$$

In $\left(c_{1}\right)-\left(c_{3}\right)$, since from $(7) \underline{e}<0,(23)$ implies that $\omega>0$ and $v^{0} \neq 0$; then, $v^{0} \in \mathscr{A}$ in $\left(\mathcal{c}_{3}\right)$, while $(24) \Rightarrow v^{0} \in \mathscr{A}$ in $\left(c_{1}\right)$ and $\left(c_{2}\right)$. In case $\left(c_{4}\right)$ (where from (8) $\underline{e} \geq 0$ ), since from (17) $\sum_{j=1}^{m} q_{j}\left(t_{1}\left(v^{k}\right)\right)^{q_{j}-p} D_{j}\left(v^{k}\right)=p$, we have $\sum_{j=1}^{m} q_{j} \omega^{q_{j}-p} D_{j}\left(v^{0}\right)=$ $p$ from which we obtain (25).

Evidently, ((24), (25)) $\Rightarrow \omega=t_{1}\left(v^{0}\right)$; then, (23) can be written in the form $\left(t_{1}\left(v^{0}\right)\right)^{p}-\sum_{j=1}^{m}\left(t_{1}\left(v^{0}\right)\right)^{q_{j}} D_{j}\left(v^{0}\right)=\underline{e}$; that is, $\widetilde{\widetilde{E}}_{1}\left(v^{0}\right)=\underline{e}$. Then, (15) holds.

Let us prove that

$$
H_{\lambda \mu}\left(v^{0}\right)=1 \text { in (19). }
$$

In fact, taking into account that $\left(i_{14}\right)$ holds in $\left(c_{1}\right)$ and $\left(i_{13}\right)$ holds in $\left(c_{2}\right)-\left(c_{4}\right)$, we obtain

$$
\begin{array}{r}
v^{0} \in \mathscr{A} \Longrightarrow \\
\left\|v^{0}\right\|>0 \Longrightarrow \\
H_{\lambda \mu}\left(v^{0}\right)>0 .
\end{array}
$$

On the other hand, since $v^{0} \in \mathscr{A} \Rightarrow \theta v^{0} \in \mathscr{A} \forall \theta>0$, we have

$$
\begin{aligned}
& \frac{d}{d \theta} \widetilde{\widetilde{E}}_{1}\left(\theta v^{0}\right)=\left[p\left(t_{1}\left(\theta v^{0}\right)\right)^{p-1}\right. \\
& \left.\quad-\sum_{j=1}^{m} q_{j}\left(t_{1}\left(\theta v^{0}\right)\right)^{q_{j}-1} D_{j}\left(\theta v^{0}\right)\right] \frac{d}{d \theta} t_{1}\left(\theta v^{0}\right) \\
& \quad-\sum_{j=1}^{m} q_{j}\left(t_{1}\left(\theta v^{0}\right)\right)^{q_{j}} \theta^{q_{j}-1} D_{j}\left(v^{0}\right) \\
& \quad=-\theta^{-1} p\left(t_{1}\left(\theta v^{0}\right)\right)^{p} \quad \forall \theta>0 .
\end{aligned}
$$

Then, if $H_{\lambda \mu}\left(v^{0}\right)<1$, we get the contradiction

$$
\begin{gathered}
\left(H_{\lambda \mu}\left(v^{0}\right)\right)^{-1 / p} v^{0} \in S_{\lambda \mu} \cap \mathscr{A}, \\
\widetilde{\widetilde{E}}_{1}\left(\left(H_{\lambda \mu}\left(v^{0}\right)\right)^{-1} v^{0}\right)<\widetilde{\widetilde{E}}_{1}\left(v^{0}\right)=\underline{e} .
\end{gathered}
$$

Relations (14), (15), and (26) allow (11).

From (11), a Lagrange multiplier $\tau$ exists such that

$$
\left\langle\partial \widetilde{\widetilde{E}}\left(v^{0}\right), v 》=\tau\left\langle\partial H_{\lambda \mu}\left(v^{0}\right), v 》 \quad \forall v \in W .\right.\right.
$$

Setting in (30) $v=v^{0}$, we get $\tau=0$, since $\left\langle\partial H_{\lambda \mu}\left(v^{0}\right), v^{0}\right\rangle=$ $p H_{\lambda \mu}\left(v^{0}\right)=p$, and from (9) $\left\langle\partial \partial \widetilde{\widetilde{E}}\left(v^{0}\right), v^{0}\right\rangle=$ $\left(t\left(v^{0}\right)\right)^{p}\left\langle\partial H_{\lambda \mu}\left(v^{0}\right), v^{0}\right\rangle-\sum_{j=1}^{m}\left(t\left(v^{0}\right)\right)^{q_{j}}\left\langle\partial \partial D_{j}\left(v^{0}\right), v^{0}\right\rangle=$ $p\left(t\left(v^{0}\right)\right)^{p}-\sum_{j=1}^{m} q_{j}\left(t\left(v^{0}\right)\right)^{q_{j}} D_{j}\left(v^{0}\right)=0$. Then, (30), taking into account (10), implies $\left\langle\partial H_{\lambda \mu}\left(t\left(v^{0}\right) v^{0}\right), v 》=\right.$ $\sum_{j=1}^{m}\left\langle\partial D_{j}\left(t\left(v^{0}\right) v^{0}\right), v 》 \forall v \in W\right.$; then, (12) holds.

Proposition 2. Let $n>1$. Let $v^{0}$ as in Theorem 1 and $\mathscr{F} \subseteq$ $S_{\lambda \mu} \cap \mathscr{A}$. Let us suppose the following:

$\left(i_{0}^{h}\right) \forall v \in \mathscr{F}_{h} \in W_{h} \backslash\{0\}$ and a function $\Phi\left(\cdot, v, \widetilde{v}_{h}\right) \rightarrow$ $W$ belonging to $\left.\left.C^{o}(] s_{0}, 1\right]\right) \cap C^{1}(] s_{0}, 1[)\left(0 \leq s_{0}<1\right)$ [resp., $C^{o}\left(\left[1, s_{0}[) \cap C^{1}(] 1, s_{0}[)\left(1<s_{0} \leq+\infty\right)\right]\right.$ exist such that $\Phi\left(1, v, \widetilde{v}_{h}\right)=v, \widetilde{v}(s)=\Phi\left(s, v, \widetilde{v}_{h}\right) \in S_{\lambda \mu} \forall s \in$ ] $\left.s_{0}, 1\right]$ [resp., $\forall s \in\left[1, s_{0}[]\right.$ and for some $\left.s_{1} \in\right] s_{0}, 1[$ $\left[\right.$ resp., $\left.s_{1} \in\right] 1, s_{0}[] \sum_{j=1}^{m}(t(\widetilde{v}(s)))^{q_{j}}(d / d s) D_{j}(\widetilde{v}(s))<0$ [resp., $>0] \forall s \in] s_{1}, 1[$ [resp., $\forall s \in] 1, s_{1}[]$.

Then, $v^{0} \notin \mathscr{F}$.

Proof. In fact, with $v$ and $\widetilde{v}(s)$ as in $\left.\left(i_{0}^{h}\right) s_{2} \in\right] s_{1}, 1\left[\right.$ [resp., $s_{2} \in$ ]1, $s_{1}[]$ exists such that $\widetilde{v}(s) \in S_{\lambda \mu} \cap \mathscr{A}$ and $(d / d s) \widetilde{\widetilde{E}}(\widetilde{v}(s))=$ $-\sum_{j=1}^{m}(t(\widetilde{v}(s)))^{q_{j}}(d / d s) D_{j}(\widetilde{v}(s))>0[$ resp., < 0$\left.] \forall s \in\right] s_{2}, 1[$ [resp., $\forall s \in] 1, s_{2}[$ ]; then, $\widetilde{\widetilde{E}}(\widetilde{v}(s))<\tilde{\widetilde{E}}(v) \forall s \in] s_{2}, 1[$ [resp., $\forall s \in] 1, s_{2}[]$.

Remark 3. Condition $\left(i_{0}^{h}\right)$ with $\mathscr{F} \subseteq S_{\lambda \mu}$ includes condition $\left(i_{16}^{h}\right)$ introduced in [10] and it implies the conclusion of Theorem 2.2 of [10]. 
In the case

$\left(c_{5}\right) m>1,\left(i_{15}\right)$ holds, $q_{m}<p$, and $D_{j} \leq 0 \forall j \in$ $\{2, \ldots, m\}$,

for each $v \in V_{\lambda \mu}^{-} \cap S\left(D_{1}\right)$, (6) has only one positive root $t(v)$ and we have $(\partial \psi / \partial t)(t(v), v) \neq 0$.

Set $\widetilde{\widetilde{E}}(v)=\widetilde{E}(t(v), v) \forall v \in V_{\lambda \mu}^{-} \cap S\left(D_{1}\right)$, in [10, Theorem 4.2], the following result has been proved.

Theorem 4. Under conditions $\left(i_{11}\right),\left(i_{12}\right)$, in case $\left(c_{5}\right)$, one has the following:

$$
\begin{gathered}
\exists \bar{v}=\left(\bar{v}_{1}, \ldots, \bar{v}_{n}\right) \in V_{\lambda \mu}^{-} \cap S\left(D_{1}\right): \\
\widetilde{\widetilde{E}}(\bar{v})=\inf \left\{\widetilde{\widetilde{E}}(v): v \in V_{\lambda \mu}^{-} \cap S\left(D_{1}\right)\right\}, \\
\bar{u}=t(\bar{v}) \bar{v} \text { is solution of Problem }(P) .
\end{gathered}
$$

We add the following proposition.

Proposition 5. Let $n>1$. Let $\bar{v}$ as in Theorem 4 and $\mathscr{F} \subseteq$ $V_{\lambda \mu}^{-} \cap S\left(D_{1}\right)$. Let one suppose the following:

$\left(\underline{i}^{h}\right) \forall v \in \mathscr{F} \widetilde{v}_{h} \in W_{h} \backslash\{0\}$ and a function $\Phi\left(\cdot, v, \widetilde{v}_{h}\right) \rightarrow$ $W$ belonging to $\left.\left.C^{o}(] s_{0}, 1\right]\right) \cap C^{1}(] s_{0}, 1[)\left(0 \leq s_{0}<\right.$ 1) [resp., $C^{o}\left(\left[1, s_{0}[) \cap C^{1}(] 1, s_{0}[)\left(1<s_{0} \leq+\infty\right)\right]\right.$ exist such that $\Phi\left(1, v, \widetilde{v}_{h}\right)=v, \widetilde{v}(s)=\Phi\left(s, v, \widetilde{v}_{h}\right) \in$ $\left.\left.S\left(D_{1}\right) \forall s \in\right] s_{0}, 1\right]$ [resp., $\forall s \in\left[1, s_{0}[]\right.$ and for some $s_{1} \in$ ]$s_{0}, 1\left[\right.$ [resp., $\left.s_{1} \in\right] 1, s_{0}[](t(\widetilde{v}(s)))^{p}(d / d s) H_{\lambda \mu}(\widetilde{v}(s))-$ $\sum_{j=1}^{m}(t(\widetilde{v}(s)))^{q_{j}}(d / d s) D_{j}(\widetilde{v}(s))>0[$ resp., $<0] \forall s \in$ ]$s_{1}, 1[$ [resp., $\forall s \in] 1, s_{1}[]$.

Then, $\bar{v} \notin \mathscr{F}$.

Proof. Let $v$ and $\widetilde{v}(s)$ be as in $\left(\underline{i}^{h}\right)$. Since $t(v)>0, \psi(t(v), v)=$ 0 and $(\partial \psi / \partial t)(t(v), v) \neq 0$; then, an open ball $\widetilde{B}$ exists with center $v$ included in $V_{\lambda \mu}^{-}$and a unique functional $t^{*}(w)$ belonging to $C^{1}(\widetilde{B})$ such that $t^{*}(w)>0$ and $\psi\left(t^{*}(w), w\right)=$ $0 \forall w \in \widetilde{B}$. Evidently, $t^{*}(w)=t(w) \forall w \in \widetilde{B} \cap S\left(D_{1}\right)$. Set $\left.s_{2} \in\right] s_{1}, 1\left[\left[\right.\right.$ resp., $\left.s_{2} \in\right] 1, s_{1}[]$ such that $\left.\left.\widetilde{v}(s) \in \widetilde{B} \forall s \in\right] s_{2}, 1\right]$ [resp., $\forall s \in\left[1, s_{2}[]\right.$; we have

$$
\begin{aligned}
& \frac{d}{d s}\left[\left(t^{*}(\widetilde{v}(s))\right)^{p} H_{\lambda \mu}(\widetilde{v}(s))\right. \\
& \left.-\sum_{j=1}^{m}\left(t^{*}(\widetilde{v}(s))\right)^{q_{j}} D_{j}(\widetilde{v}(s))\right] \\
& =(t(\widetilde{v}(s)))^{p} \frac{d}{d s} H_{\lambda \mu}(\widetilde{v}(s)) \\
& -\sum_{j=1}^{m}(t(\widetilde{v}(s)))^{q_{j}} \frac{d}{d s} D_{j}(\widetilde{v}(s))>0 \\
& \quad[\text { resp., }<0] \forall s \in] s_{2}, 1\left[[\text { resp. }, \forall s \in] 1, s_{2}[] .\right.
\end{aligned}
$$

Consequently, $\widetilde{\widetilde{E}}(\widetilde{v}(s))<\widetilde{\widetilde{E}}(v) \forall s \in] s_{2}, 1[$ [resp., $\forall s \in] 1, s_{2}[]$.
Remark 6. Let $W_{\ell}(\ell=1, \ldots, n)$ be a vector lattice. Let $v^{0}$ [resp., $\bar{v}$ ] be as in Theorem 1 [resp., Theorem 4]. If $H_{\lambda \mu}\left(v_{1}, \ldots, v_{n}\right)=H_{\lambda \mu}\left(\left|v_{1}\right|, \ldots,\left|v_{n}\right|\right)$ and $D_{j}\left(v_{1}, \ldots, v_{n}\right)=$ $D_{j}\left(\left|v_{1}\right|, \ldots,\left|v_{n}\right|\right) \forall v=\left(v_{1}, \ldots, v_{n}\right) \in W$ and $\forall j \in\{1, \ldots, m\}$, then $\left(\left|v_{1}^{0}\right|, \ldots,\left|v_{n}^{0}\right|\right) \in S_{\lambda \mu} \cap \mathscr{A}, t\left(\left|v_{1}^{0}\right|, \ldots,\left|v_{n}^{0}\right|\right)=t\left(v^{0}\right)$, $\widetilde{\widetilde{E}}\left(\left|v_{1}^{0}\right|, \ldots,\left|v_{n}^{0}\right|\right)=\widetilde{\widetilde{E}}\left(v^{0}\right)\left[\operatorname{resp} .,\left(\left|\bar{v}_{1}\right|, \ldots,\left|\bar{v}_{n}\right|\right) \in V_{\lambda \mu}^{-} \cap S\left(D_{1}\right)\right.$, $\left.t\left(\left|\bar{v}_{1}\right|, \ldots,\left|\bar{v}_{n}\right|\right)=t(\bar{v}), \widetilde{\widetilde{E}}\left(\left|\bar{v}_{1}\right|, \ldots,\left|\bar{v}_{n}\right|\right)=\widetilde{\widetilde{E}}(\bar{v})\right]$.

Then, reasoning as in Theorem 1 proof's final step [resp., Theorem 4.2 of [10]], we see that $\pm t\left(v^{0}\right)\left(\left|v_{1}^{0}\right|, \ldots,\left|v_{n}^{0}\right|\right)$ [resp., \pm $\left.t(\bar{v})\left(\left|\bar{v}_{1}\right|, \ldots,\left|\bar{v}_{n}\right|\right)\right]$ are solutions of Problem (P). Consequently, we can assume that $v_{\ell}^{0} \geq 0$; that is, $u_{\ell}^{0} \geq 0$ [resp., $\bar{v}_{\ell} \geq 0$ i.e., $\bar{u}_{\ell} \geq 0$ ] as $\ell=1, \ldots, n$.

\section{Dirichlet Problems}

We assume $W=\left(W_{0}^{1, p}(\Omega)\right)^{n}(n \geq 1)$ with $\|v\|=$ $\left(\sum_{\ell=1}^{n} \int_{\Omega}\left|\nabla v_{\ell}\right|^{p} d x\right)^{1 / p} \forall v=\left(v_{1}, \ldots, v_{n}\right) \in W, B_{\ell}\left(v_{\ell}\right)=$ $p^{-1} \int_{\Omega} b_{\ell}\left|v_{\ell}\right|^{p} d x \forall v_{\ell} \in W_{0}^{1, p}(\Omega)$, and $\widehat{B}_{\ell} \equiv 0$, where $1<$ $p<\infty, \Omega \subseteq R^{N}$ is open, bounded, connected and $C^{2, \beta}$ set $(0<\beta \leq 1), b_{\ell} \in L^{\infty}(\Omega) \backslash\{0\}$ with $b_{\ell} \geq 0$.

Let us consider the functional $A$ (as in $\left(i_{11}\right)$ ) such that $A(v) \geq p^{-1} \widetilde{c}\|v\|^{p} \forall v \in W(\widetilde{c}=$ const. $>0)$.

Let us use the following notations: $\widetilde{p}=N p /(N-p)$ if $N>$ $p, \tilde{p}=\infty$ else; $|\cdot|_{N}$ is the Lebesgue measure in $R^{N} ; H_{\lambda}, S_{\lambda}$, $V_{\lambda}^{-}$are instead of $H_{\lambda \mu}, S_{\lambda \mu}, V_{\lambda \mu}^{-} ; \lambda_{\ell}^{*}$ is the first eigenvalue and $u_{\ell}^{*}$ is the first eigenfunction of the problem $u_{\ell} \in W_{0}^{1, p}(\Omega)$ : $-\widetilde{c} \operatorname{div}\left(\left|\nabla u_{\ell}\right|^{p-2} \nabla u_{\ell}\right)=\theta b_{\ell}\left|u_{\ell}\right|^{p-2} u_{\ell}$ in $\Omega$ [16].

We present some results about the validity of assumptions $\left(i_{13}\right)-\left(i_{15}\right)$. To this aim, let us set $I=\{1, \ldots, n\}$ and, for each $I^{*}(\neq \varnothing) \subseteq I, V^{*}=\left\{v \in W: v_{\ell} \equiv 0\right.$ if $\ell \in I \backslash I^{*}, v_{\ell}=$ $c_{\ell} u_{\ell}^{*}$ if $\ell \in I^{*}$ with $c_{\ell} \in R$ and $c_{\ell} \neq 0$ as some $\left.\ell \in I^{*}\right\}$, let us introduce the following hypothesis:

$\left(i_{3}\right) I^{*} \subseteq I$ exists: $D_{1}(v)<0$ for every $v \in V^{*}$.

$\left(\underline{i}_{3}\right) I^{*} \subseteq I$ exists such that $D_{1}(v)<0$ and $A(v)=$ $\widetilde{c} p^{-1} \sum_{\ell \in I^{*}} \int_{\Omega}\left|\nabla v_{\ell}\right|^{p} d x$ for every $v \in V^{*}$.

Proposition 7. If $\lambda_{\ell}<\lambda_{\ell}^{*}$ for each $\ell \in\{1, \ldots, n\}$, then $\left(i_{13}\right)$ holds. When $V^{+}\left(D_{1}\right) \neq \varnothing$ and $\left(i_{3}\right)$ holds with $I^{*} \neq I$, then, with $\lambda_{\ell}<\lambda_{\ell}^{*}$ as $\ell \in I \backslash I^{*}, \exists \delta^{*}>0:\left(i_{14}\right)$ holds if $\lambda_{\ell}<\lambda_{\ell}^{*}+\delta^{*}$ as $\ell \in I^{*}$. When $V^{+}\left(D_{1}\right) \neq \varnothing$ and $\left(i_{3}\right)$ holds with $I^{*}=I$, then $\exists \delta^{*}>0:\left(i_{14}\right)$ holds if $\lambda_{\ell}<\lambda_{\ell}^{*}+\delta^{*}$ as $\ell \in I$.

Proof. The first statement is obvious. We can prove the second and the third ones as in [11, Propositions 2.3 and 2.4].

Proposition 8. When $\left(\underline{i}_{3}\right)$ holds with $I^{*} \neq I$, then with $\lambda_{\ell}<$ $\lambda_{\ell}^{*}$ as $\ell \in I \backslash I^{*} \exists \delta^{*}>0:\left(i_{15}\right)$ holds if $\lambda_{\ell} \in\left[\lambda_{\ell}^{*}, \lambda_{\ell}^{*}+\delta^{*}[\forall \ell \in\right.$ $I^{*}$ and $\lambda_{\ell}>\lambda_{\ell}^{*}$ as some $\ell$. When $\left(i_{3}\right)$ holds with $I^{*}=I$, then $\exists \delta^{*}>0:\left(i_{15}\right)$ holds if $\lambda_{\ell} \in\left[\lambda_{\ell}^{*}, \lambda_{\ell}^{*}+\delta^{*}\left[\forall \ell \in I\right.\right.$ and $\lambda_{\ell}>\lambda_{\ell}^{*}$ as some $\ell$.

Proof. See [11, Propositions 2.5 and 2.6]. 
Let us now investigate Problem $(\mathrm{P})$ in two concrete cases where $\widetilde{c}=1$.

Application 1. Let $n>1$ and, for each $v=\left(v_{1}, \ldots, v_{n}\right) \in W$,

$$
\begin{aligned}
& A(v)=p^{-1} \int_{\Omega}\left(\sum_{\ell=1}^{n}\left|\nabla v_{\ell}\right|^{\gamma}\right)^{p / \gamma} d x, \\
& D_{1}(v)=\left(\int_{\Omega} \rho_{1}\left|v_{1}\right|^{q_{11}} d x\right) \int_{\Omega}\left(\sum_{\ell=1}^{n}\left|v_{\ell}\right|^{\gamma_{1}}\right)^{q_{12} / \gamma_{1}} d x, \\
& D_{j}(v)=\left.q_{j}^{-1} \int_{\Omega} \rho_{j}\left|d_{j 1}\right| v_{1}\right|^{\gamma_{j}}+\left.d_{j j}\left|v_{j}\right|^{\gamma_{j}}\right|^{q_{j} / \gamma_{j}} d x \\
& \text { as } j=2, \ldots, n,
\end{aligned}
$$

where

$$
\begin{aligned}
& 1<\gamma<p, \\
& q_{11}>1, \\
& 1<\gamma_{1}<q_{12}, \\
& 1<\gamma_{j}<q_{j} \\
& \text { as } j=2, \ldots, n, q_{1}=q_{11}+q_{12}<q_{2}<\cdots<q_{n}<\tilde{p}, q_{1}<p ; \\
& \rho_{1} \in L^{\infty}(\Omega) ; \\
& \rho_{j} \in L^{\infty}(\Omega), \\
& \rho_{j}<0, \\
& d_{j 1}, d_{j j} \in L^{\infty}(\Omega) \backslash\{0\}
\end{aligned}
$$

Let us consider the following system:

$$
\begin{aligned}
& -\operatorname{div}\left[\left(\sum_{\ell=1}^{n}\left|\nabla u_{\ell}\right|^{\gamma}\right)^{(p / \gamma)-1}\left|\nabla u_{1}\right|^{\gamma-2} \nabla u_{1}\right] \\
& =\lambda_{1} b_{1}\left|u_{1}\right|^{p-2} u_{1} \\
& +q_{11}\left(\int_{\Omega}\left(\sum_{\ell=1}^{n}\left|u_{\ell}\right|^{\gamma_{1}}\right)^{\left(q_{12} / \gamma_{1}\right)} d x\right) \rho_{1}\left|u_{1}\right|^{q_{11}-2} u_{1} \\
& +q_{12}\left(\int_{\Omega} \rho_{1}\left|u_{1}\right|^{q_{11}} d x\right)\left(\sum_{\ell=1}^{n}\left|u_{\ell}\right|^{\gamma_{1}}\right)^{\left(q_{12} / \gamma_{1}\right)-1} \\
& \cdot\left|u_{1}\right|^{\gamma_{1}-2} u_{1}+\left.\sum_{j=2}^{n} \rho_{j}\left|d_{j 1}\right| u_{1}\right|^{\gamma_{j}}+\left.d_{j j}\left|u_{j}\right|^{\gamma_{j}}\right|^{\left(q_{j} / \gamma_{j}\right)-2} \\
& \cdot\left(d_{j 1}\left|u_{1}\right|^{\gamma_{j}}+d_{j j}\left|u_{j}\right|^{\gamma_{j}}\right) d_{j 1}\left|u_{1}\right|^{\gamma_{j}-2} u_{1}
\end{aligned}
$$

$$
\begin{aligned}
& -\operatorname{div}\left[\left(\sum_{\ell=1}^{n}\left|\nabla u_{\ell}\right|^{\gamma}\right)^{(p / \gamma)-1}\left|\nabla u_{i}\right|^{\gamma-2} \nabla u_{i}\right] \\
& =\lambda_{i} b_{i}\left|u_{i}\right|^{p-2} u_{i}+q_{12}\left(\int_{\Omega} \rho_{1}\left|u_{1}\right|^{q_{11}} d x\right)\left(\sum_{\ell=1}^{n}\left|u_{\ell}\right|^{\gamma_{1}}\right)^{\left(q_{12} / \gamma_{1}\right)-1} \\
& \cdot\left|u_{i}\right|^{\gamma_{1}-2} u_{i}+\left.\rho_{i}\left|d_{i 1}\right| u_{1}\right|^{\gamma_{i}}+\left.d_{i i}\left|u_{i}\right|^{\gamma_{i}}\right|^{\left(q_{i} / \gamma_{i}\right)-2} \\
& \cdot\left(d_{i 1}\left|u_{1}\right|^{\gamma_{i}}+d_{i i}\left|u_{i}\right|^{\gamma_{i}}\right) d_{i i}\left|u_{i}\right|^{\gamma_{i}-2} u_{i} \\
& \quad \text { in } \Omega \text { as } i=2, \ldots, n, \\
& u_{i}=0 \quad \text { on } \partial \Omega \text { as } i=1, \ldots, n .
\end{aligned}
$$

Let us introduce the following conditions:

$$
\rho_{1}^{+} \neq \equiv 0\left(\Longrightarrow V^{+}\left(D_{1}\right) \neq \varnothing\right) \text {, }
$$

$$
\begin{aligned}
& \int_{\Omega} \rho_{1}\left(u_{1}^{*}\right)^{q_{11}} d x \\
& \quad<0\left(\Longrightarrow D_{1}\left(c_{1} u_{1}^{*}, \ldots, c_{n} u_{n}^{*}\right)<0 \text { iff } c_{1} \neq 0\right) .
\end{aligned}
$$

Then (Propositions 7 and 8),

$(36) \Longrightarrow$

(with $\lambda_{\ell}<\lambda_{\ell}^{*}$ as $\ell=1, \ldots, n\left(i_{14}\right)$ holds),

(36), (37) $\Longrightarrow$

(with $\lambda_{\ell}<\lambda_{\ell}^{*}$ as $\ell=2, \ldots, n \exists \delta_{1}^{*}$

$$
\left.>0:\left(i_{14}\right) \text { holds if } \lambda_{1}<\lambda_{1}^{*}+\delta_{1}^{*}\right) \text {, }
$$

(37) $\Longrightarrow$

(with $\lambda_{\ell}<\lambda_{\ell}^{*}$ as $\ell=2, \ldots, n \exists \delta_{2}^{*}$

$$
\left.>0:\left(i_{15}\right) \text { holds if } \lambda_{1} \in\right] \lambda_{1}^{*}, \lambda_{1}^{*}+\delta_{2}^{*}[) \text {. }
$$

Proposition 9 (Theorems 1 and 4, Remark 6,). Under conditions (34), one has the following:

When (36) holds [resp., (36) and (37) hold], with $\lambda_{1}, \ldots, \lambda_{n}$ as in (38) [resp., (39)], system (35) has at least two weak solutions $u^{0}$ and $-u^{0}\left(u^{0}=\tau^{0} v^{0}, \tau^{0}=\right.$ const. $\left.>0, v^{0} \in S_{\lambda} \cap V^{+}\left(D_{1}\right)\right)$, and one has $u_{i}^{0} \geq 0$ as $i=1, \ldots, n, u_{1}^{0} \not \equiv 0$.

When $q_{n}<p$ and (37) holds, with $\lambda_{1}, \ldots, \lambda_{n}$ as in (40), system (35) has at least two weak solutions $\bar{u}$ and $-\bar{u}\left(\bar{u}=\bar{\tau} v, \bar{\tau}=\right.$ const. $\left.>0, \bar{v} \in V_{\lambda}^{-} \cap S\left(D_{1}\right)\right)$, and one has $\bar{u}_{i} \geq 0$ as $i=1, \ldots, n, \bar{u}_{1} \neq \equiv$.

Consequently, when $q_{n}<p$ and (36), (37) hold, with $\lambda_{\ell}<\lambda_{\ell}^{*}$ as $\ell \geq 2$ and $\left.\lambda_{1} \in\right] \lambda_{1}^{*}, \lambda_{1}^{*}+\min \left\{\delta_{1}^{*}, \delta_{2}^{*}\right\}[$, system (35) has at least four different weak solutions. 
Proposition 10. If

$$
\begin{gathered}
\text { either } \gamma_{1}<\gamma \leq \gamma_{j} \text { as } j=2, \ldots, n \\
\text { or } d_{j 1} d_{j j}<0 \\
\gamma_{j}<\gamma
\end{gathered}
$$

as $j=2, \ldots, n$,

then $u_{i}^{0} \not \equiv 0$ as $i=2, \ldots, n$.

Proof. It is sufficient (Proposition 2) to verify the following. As $h=2, \ldots, n\left(i_{0}^{h}\right)$ holds with $\mathscr{F}=\left\{v \in S_{\lambda} \cap V^{+}\left(D_{1}\right)\right.$ : $v_{\ell} \geq 0$ as $\left.\ell=1, \ldots, n, v_{1} \not \equiv 0, v_{h} \equiv 0\right\} \neq \varnothing$.

Let, for example, $h=2$. Let $v \in \mathscr{F}$. First of all,

$$
\begin{aligned}
& \int_{\Omega} \rho_{1} v_{1}^{q_{11}} d x>0, \\
& \lambda_{2}<\lambda_{2}^{*} \Longrightarrow \\
& \delta=\int_{\Omega}\left|\nabla v_{1}\right|^{p} d x-\lambda_{2} \int_{\Omega} b_{2} v_{1}^{p} d x>0 .
\end{aligned}
$$

Let us introduce the function $g(s, \tau)=H_{\lambda}\left(s v_{1}, \tau v_{1}, \ldots, s v_{n}\right)$ $=p^{-1}\left[\int_{\Omega}\left(\tau^{\gamma}\left|\nabla v_{1}\right|^{\gamma}+s^{\gamma} \sum_{\ell \neq 2}\left|\nabla v_{\ell}\right|^{\gamma}\right)^{p / \gamma} d x-\tau^{p} \lambda_{2} \int_{\Omega} b_{2} v_{1}^{p} d x-\right.$ $\left.s^{p} \sum_{\ell \neq 2} \lambda_{\ell} \int_{\Omega} b_{\ell} v_{\ell}^{p} d x\right] \forall s, \tau \geq 0$. Let us note that

$$
\begin{aligned}
g(1,0) & =1, \\
\frac{\partial g}{\partial \tau}(s, \tau) & \geq \delta \tau^{p-1}>0 \quad \forall s \geq 0, \quad \forall \tau>0, \\
g(s, 0) & \left.=s^{p}<1 \quad \forall s \in\right] 0,1[, \\
\lim _{\tau \rightarrow+\infty} g(s, \tau) & =+\infty \quad \forall s \geq 0 ;
\end{aligned}
$$

then,

only one $\tau(s)>0$

$$
\begin{aligned}
(\tau(s) & >0 \text { if } s<1, \tau(1)=0) \text { exists such that } \\
g(s, \tau(s)) & =1,
\end{aligned}
$$

$$
\forall s \in] 0,1]
$$

About the function $\tau(s)$, we have

$$
\lim _{\tau \rightarrow 1^{-}} \tau(s)=0 .
$$

In fact, with $\left.\left\{s_{n}\right\} \subseteq\right] 0,1\left[\right.$ and $\lim s_{n}=1$, since $g\left(s_{n}, \tau\left(s_{n}\right)\right)=1$, $\left\{\tau\left(s_{n}\right)\right\}$ is necessarily bounded. Then (within a subsequence), $\lim \tau\left(s_{n}\right)=\omega$ with $g(1, \omega)=1$, from which $\omega=0$. Then, $\tau(s)$ belongs to $\left.\left.C^{0}(] 0,1\right]\right)$.

Let us add that $\tau(s)$ belongs to $C^{1}(] 0,1[)$ and it results in

$$
\begin{aligned}
& \tau^{\prime}(s)=-\frac{1}{(\tau(s))^{\gamma-1}} \tilde{g}(s, \tau(s)) \\
&\quad \forall s \in] 0,1\left[, \lim _{s \rightarrow 1^{-}} \tilde{g}(s, \tau(s)) \in\right] 0,+\infty[.
\end{aligned}
$$

Then, with $\widetilde{v}(s)=\left(s v_{1}, \tau(s) v_{1}, \ldots, s v_{n}\right)$, we have

$$
\begin{aligned}
& \widetilde{v}(1)=v, \\
& \left.\left.\widetilde{v}(s) \in S_{\lambda} \quad \forall s \in\right] 0,1\right],
\end{aligned}
$$

and, moreover, taking into account the first one of (43),

$$
\begin{aligned}
& \lim _{s \rightarrow 1^{-}} \frac{d}{d s} D_{1}(\widetilde{v}(s))=-\infty, \\
& \lim _{s \rightarrow 1^{-}} \frac{d}{d s} D_{j}(\widetilde{v}(s)) \in R \text { as } j \geq 2 \text { when (41) holds, } \\
& \lim _{s \rightarrow 1^{-}} \frac{d}{d s} D_{1}(\widetilde{v}(s))<+\infty, \\
& \lim _{s \rightarrow 1^{-}} \frac{d}{d s} D_{2}(\widetilde{v}(s))=-\infty,
\end{aligned}
$$$$
\lim _{s \rightarrow 1^{-}} \frac{d}{d s} D_{j}(\widetilde{v}(s)) \in R
$$$$
\text { as } j>2 \text { (if } n>2 \text { ) when (42) holds, }
$$

from which $\lim _{s \rightarrow 1^{-}} \sum_{j=1}^{n}(t(\widetilde{v}(s)))^{q_{j}}(d / d s) D_{j}(\widetilde{v}(s))=-\infty$.

Proposition 11. If $d_{j 1} d_{j j}<0$ and $\gamma_{j}<\gamma_{1} \leq \gamma$ as $j=2, \ldots, n$, then $\bar{u}_{i} \not \equiv 0$ as $i=2, \ldots, n$.

Proof. It is sufficient (Proposition 5) to prove the following.

As $h=2, \ldots, n\left(\underline{i}^{h}\right)$ holds with $\mathscr{F}=\left\{v \in V_{\lambda}^{-} \cap S\left(D_{1}\right)\right.$ : $v_{\ell} \geq 0$ as $\left.\ell=1, \ldots, n, v_{1} \not \equiv 0, v_{h} \equiv 0\right\} \neq \varnothing$.

Let, for example, $h=2$. Let $v \in \mathscr{F}$. Firstly, we have

$$
\int_{\Omega} \rho_{1} v_{1}^{q_{11}} d x<0
$$

Let us consider the function $g_{1}(s, \tau)=D_{1}\left(s v_{1}, \tau v_{1}, \ldots, s v_{n}\right)=$ $s^{q_{11}}\left(\int_{\Omega} \rho_{1} v_{1}^{q_{11}} d x\right) \int_{\Omega}\left(\tau^{\gamma_{1}} v_{1}^{\gamma_{1}}+s^{\gamma_{1}} \sum_{\ell \neq 2} v_{\ell}^{\gamma_{1}}\right)^{q_{12} / \gamma_{1}} d x \forall s, \tau \geq 0$. Since

$$
\begin{aligned}
g_{1}(1,0) & =-1, \\
\frac{\partial g_{1}}{\partial \tau}(s, \tau) & <0 \quad \forall s>0, \forall \tau>0, \\
g_{1}(s, 0) & \left.=-s^{q_{1}}>-1 \quad \forall s \in\right] 0,1[, \\
\lim _{\tau \rightarrow+\infty} g_{1}(s, \tau) & =-\infty \quad \forall s>0,
\end{aligned}
$$


we have the following:

only one $\tau(s) \geq 0$

$$
\begin{aligned}
(\tau(s) & >0 \text { if } s<1, \tau(1)=0) \text { exists such that } \\
g_{1}(s, \tau(s)) & =-1,
\end{aligned}
$$

$\forall s \in] 0,1]$

the function $\tau(s)$ belongs to $\left.\left.C^{0}(] 0,1\right]\right) \cap C^{1}(] 0,1[), \tau^{\prime}(s)=$ $-\left(1 /(\tau(s))^{\gamma_{1}-1}\right) \widetilde{g}_{1}(s, \tau(s))$, and

$$
\left.\lim _{s \rightarrow 1^{-}} \widetilde{g}_{1}(s, \tau(s)) \in\right] 0,+\infty[, \quad \forall s \in] 0,1[
$$

Then, set $\widetilde{v}(s)=\left(s v_{1}, \tau(s) v_{1}, \ldots, s v_{n}\right)$; we get

$$
\begin{aligned}
\widetilde{v}(1) & =v, \\
D_{1}(\widetilde{v}(s)) & =-1 \quad \forall s \in] 0,1], \\
\lim _{s \rightarrow 1^{-}} \frac{d}{d s} H_{\lambda}(\widetilde{v}(s)) & \in R, \\
\lim _{s \rightarrow 1^{-}} \frac{d}{d s} D_{2}(\widetilde{v}(s)) & =-\infty, \\
\lim _{s \rightarrow 1^{-}} \frac{d}{d s} D_{j}(\widetilde{v}(s)) & \in R \quad \text { as } j>2 \text { (if } n>2) ;
\end{aligned}
$$

in particular, $\quad \lim _{s \rightarrow 1^{-}}\left[(t(\widetilde{v}(s)))^{p}(d / d s) H_{\lambda}(\widetilde{v}(s))\right.$ $\left.\sum_{j=1}^{n}(t(\widetilde{v}(s)))^{q_{j}}(d / d s) D_{j}(\widetilde{v}(s))\right]=+\infty$.

Application 2. Let $n>2$ and, for each $v=\left(v_{1}, \ldots, v_{n}\right) \in W$,

$$
\begin{aligned}
& A(v)=p^{-1} \sum_{\ell=1}^{n} \int_{\Omega}\left|\nabla v_{\ell}\right|^{p} d x, \\
& D_{1}(v)=q_{1}^{-1}\left[\int_{\Omega}\left(d_{1}\left|v_{1}\right|^{\gamma_{1}}+d \sum_{\ell=2}^{n}\left|v_{\ell}\right|^{\gamma_{1}}\right)^{q_{1} / \gamma_{1}} d x\right. \\
& \left.-\int_{\Omega} \tilde{d}\left(\sum_{\ell=2}^{n}\left|v_{\ell}\right|^{\gamma_{1}}\right)^{q_{1} / \gamma_{1}} d x\right], \\
& D_{j}(v)=\left.q_{j}^{-1} \sum_{\substack{\ell=1 \\
\ell \neq j}}^{n} \int_{\Omega}\left|d_{j j}\right| v_{j}\right|^{\gamma_{j}}+\left.d_{j \ell}\left|v_{\ell}\right|^{\gamma_{j}}\right|^{q_{j} / \gamma_{j}} d x
\end{aligned}
$$

as $j=2, \ldots, n$, where

$$
\begin{aligned}
& 1<\gamma_{j}<q_{j} \quad \text { as } j=1, \ldots, n, \\
& q_{1}<\cdots<q_{n}, \\
& q_{1}<p, \\
& q_{n}<\tilde{p} ; \\
& d_{1}, d, \tilde{d} \in L^{\infty}(\Omega), \quad \text { with } d_{1}, d>0, d^{q_{1} / \gamma_{1}}<\tilde{d} ; \\
& \rho_{j} \in L^{\infty}(\Omega), \quad \text { with } \rho_{j}<0, \\
& d_{j \ell} \in L^{\infty}(\Omega) \backslash\{0\} \quad \text { as } j=2, \ldots, n, \ell=1, \ldots, n .
\end{aligned}
$$

Let us consider the system:

$$
\begin{aligned}
& -\operatorname{div}\left[\left|\nabla u_{1}\right|^{p-2} \nabla u_{1}\right]=\lambda_{1} b_{1}\left|u_{1}\right|^{p-2} u_{1}+d_{1}\left(d_{1}\left|u_{1}\right|^{\gamma_{1}}\right. \\
& \left.+d \sum_{\ell=2}^{n}\left|u_{\ell}\right|^{\gamma_{1}}\right)^{\left(q_{1} / \gamma_{1}\right)-1}\left|u_{1}\right|^{\gamma_{1}-2} u_{1}+\left.\sum_{j=2}^{n} \rho_{j}\left|d_{j j}\right| u_{j}\right|^{\gamma_{j}} \\
& +\left.d_{j 1}\left|u_{1}\right|^{\gamma_{j}}\right|^{\left(q_{j} / \gamma_{j}\right)-2}\left(d_{j j}\left|u_{j}\right|^{\gamma_{j}}\right. \\
& \left.+d_{j 1}\left|u_{1}\right|^{\gamma_{j}}\right) d_{j 1}\left|u_{1}\right|^{\gamma_{j}-2} u_{1} \\
& -\operatorname{div}\left[\left|\nabla u_{i}\right|^{p-2} \nabla u_{i}\right]=\lambda_{i} b_{i}\left|u_{i}\right|^{p-2} u_{i}+d\left(d_{1}\left|u_{1}\right|^{\gamma_{1}}\right. \\
& \left.+d \sum_{\ell=2}^{n}\left|u_{\ell}\right|^{\gamma_{1}}\right)^{q_{1} / \gamma_{1}-1}\left|u_{i}\right|^{\gamma_{1}-2} u_{i}-\tilde{d}\left(\sum_{\ell=2}^{n}\left|u_{\ell}\right|^{\gamma_{1}}\right)^{q_{1} / \gamma_{1}-1} \\
& \cdot\left|u_{i}\right|^{\gamma_{1}-2} u_{i}+\left.\sum_{\substack{\ell=1 \\
\ell \neq i}}^{n} \rho_{i}\left|d_{i i}\right| u_{i}\right|^{\gamma_{i}}+\left.d_{i \ell}\left|u_{\ell}\right|^{\gamma_{i}}\right|^{\left(q_{i} / \gamma_{i}\right)-2} \\
& \cdot\left(d_{i i}\left|u_{i}\right|^{\gamma_{i}}+d_{i \ell}\left|u_{\ell}\right|^{\gamma_{i}}\right) d_{i i}\left|u_{i}\right|^{\gamma_{i}-2} u_{i} \\
& +\left.\sum_{\substack{j=2 \\
j \neq i}}^{n} \rho_{j}\left|d_{j j}\right| u_{j}\right|^{\gamma_{j}}+\left.d_{j i}\left|u_{i}\right|^{\gamma_{j}}\right|^{\left(q_{j} / \gamma_{j}\right)-2}\left(d_{j j}\left|u_{j}\right|^{\gamma_{j}}\right. \\
& \left.+d_{j i}\left|u_{i}\right|^{\gamma_{j}}\right) d_{j i}\left|u_{i}\right|^{\gamma_{j}-2} u_{i} \\
& \text { in } \Omega \text { as } i=2, \ldots, n \text {, } \\
& u_{i}=0 \quad \text { on } \partial \Omega \text { as } i=1, \ldots, n \text {. }
\end{aligned}
$$

Evidently, $V^{+}\left(D_{1}\right) \neq \varnothing$ and $D_{1}\left(0, c_{2} u_{2}^{*}, \ldots, c_{n} u_{n}^{*}\right)<$ $0 \forall\left(c_{2}, \ldots, c_{n}\right) \in R^{n-1} \backslash\{0\}$. Then, set $I^{*}(\neq \varnothing) \subseteq\{2, \ldots, n\}$; we have (Propositions 7 and 8 ) 
with $\lambda_{\ell}<\lambda_{\ell}^{*}$ as $\ell \in I \backslash I^{*} \exists \delta_{1}^{*}>0:\left(i_{14}\right)$ holds if $\lambda_{\ell}<\lambda_{\ell}^{*}+\delta_{1}^{*} \forall \ell \in I^{*}$,

with $\lambda_{\ell}<\lambda_{\ell}^{*}$ as $\ell \in I \backslash I^{*} \exists \delta_{2}^{*}>0:\left(i_{15}\right)$ holds if $\lambda_{\ell} \in\left[\lambda_{\ell}^{*}, \lambda_{\ell}^{*}+\delta_{2}^{*}\left[\forall \ell \in I^{*}, \lambda_{\ell}>\lambda_{\ell}^{*}\right.\right.$ as some $\ell$.

Proposition 12 (see Theorems 1 and 4, Remark 6). Under conditions (56), one has the following:

With $\lambda_{1}, \ldots, \lambda_{n}$ as in (58), system (57) has at least two weak solutions $u^{0}$ and $-u^{0}\left(u^{0}=\tau^{0} v^{0}, \tau^{0}=\right.$ const. $>$ $\left.0, v^{0} \in S_{\lambda} \cap V^{+}\left(D_{1}\right)\right)$, and one has $u_{i}^{0} \geq 0$ as $i=$ $1, \ldots, n, u_{1}^{0} \not \equiv 0$.

When $q_{n}<p$, with $\lambda_{1}, \ldots, \lambda_{n}$ as in (59), system (57) has at least two weak solutions $\bar{u}$ and $-\bar{u}(\bar{u}=\bar{\tau} v, \bar{\tau}=$ const. $\left.>0, \bar{v} \in V_{\lambda}^{-} \cap S\left(D_{1}\right)\right)$, and one has $\bar{u}_{i} \geq 0$ as $i=1, \ldots, n$.

Consequently, when $q_{n}<p$, with $\lambda_{\ell}<\lambda_{\ell}^{*}$ as $\ell \in I \backslash I^{*}, \lambda_{\ell} \in$ $\left[\lambda_{\ell}^{*}, \lambda_{\ell}^{*}+\min \left\{\delta_{1}^{*}, \delta_{2}^{*}\right\}\left[\forall \ell \in I^{*}\right.\right.$, and $\lambda_{\ell}>\lambda_{\ell}^{*}$ as some $\ell$, system (57) has at least four different weak solutions.

Proposition 13. Let $d_{j j} d_{j \ell}<0$ as $j=2, \ldots, n$ and $\ell=$ $1, \ldots, n$ with $\ell \neq j$. Let $\gamma_{j}<\gamma_{1}$ as $j=2, \ldots, n$. Then,

$$
\begin{aligned}
& u_{i}^{0} \neq \equiv 0 \text { as } i=2, \ldots, n \\
& \bar{u}_{i} \neq 0 \quad \text { as } i=1, \ldots, n .
\end{aligned}
$$

Proof. It is sufficient (Propositions 2 and 5) to prove that

As $h=2, \ldots, n\left(i_{0}^{h}\right)$ holds with $\mathscr{F}=\left\{v \in S_{\lambda}\right.$

$$
\begin{aligned}
& \cap V^{+}\left(D_{1}\right): v_{\ell} \geq 0 \text { as } \ell=1, \ldots, n, v_{1} \neq \equiv, v_{h} \\
& \equiv 0\} \neq \varnothing ;
\end{aligned}
$$

$\left(\underline{i}^{1}\right)$ holds with $\mathscr{F}=\left\{v \in V_{\lambda}^{-} \cap S\left(D_{1}\right): v_{\ell} \geq 0\right.$ as $\ell$

$$
\left.=1, \ldots, n, v_{1} \equiv 0\right\} \neq \varnothing ;
$$

As $h=2, \ldots, n\left(\underline{i}^{h}\right)$ holds with $\mathscr{F}=\left\{v \in V_{\lambda}^{-}\right.$

$$
\begin{array}{r}
\left.\cap S\left(D_{1}\right): v_{\ell} \geq 0 \text { as } \ell=1, \ldots, n, v_{1} \neq 0, v_{h} \equiv 0\right\}, \\
\text { if } \mathscr{F} \neq \varnothing .
\end{array}
$$

About (61), let $v \in \mathscr{F}$. Let $K \subseteq \Omega$ be a compact set such that $|K|_{N}>0$ and $v_{1}>0$ in $K$. Let [11, Proposition A.1] $\left(\varphi_{\varepsilon}\right)_{0<\varepsilon<\varepsilon_{0}} \subseteq C_{0}^{\infty}(\Omega)$ with $0 \leq \varphi_{\varepsilon} \leq 1$ such that

$$
\begin{aligned}
\varphi_{\varepsilon} & \longrightarrow \chi \quad \text { strongly in } L^{s}(\Omega), \\
\int_{\Omega}\left|\nabla \varphi_{\varepsilon}\right|^{s} d x & \longrightarrow+\infty
\end{aligned}
$$

where $\chi$ is the characteristic function of $K$. We choose $\varepsilon$ such that

$$
\begin{gathered}
\delta=p^{-1}\left[\int_{\Omega}\left|\nabla \varphi_{\varepsilon}\right|^{p} d x-\lambda_{h} \int_{\Omega} b_{h} \varphi_{\varepsilon}^{p} d x\right]>0 \\
\int_{\Omega} \rho_{h}\left|d_{h 1}\right|^{\left(q_{h} / \gamma_{h}\right)-2} v_{1}^{q_{h}-\gamma_{h}} d_{h 1} d_{h h} \varphi_{\varepsilon}^{\gamma_{h}} d x>0
\end{gathered}
$$

and let us set $\widetilde{v}_{h}=\delta^{-1 / p} \varphi_{\varepsilon}$. With $\widetilde{v}(s)=\left(s^{1 / p} v_{1}, \ldots,(1-\right.$ $\left.s)^{1 / p} \widetilde{v}_{h}, \ldots, s^{1 / p} v_{n}\right)$, we have

$$
\begin{aligned}
& \widetilde{v}(1)=v, \\
& \widetilde{v}(s) \in S_{\lambda} \quad \forall s \in[0,1] .
\end{aligned}
$$

It is easy to verify that

$$
\frac{d}{d s} D_{1}(\widetilde{v}(s))=-(1-s)^{\gamma_{1} / p-1} f_{1}(s)+\widetilde{f}_{1}(s)
$$$$
\forall s \in\left[0,1\left[\text { with } \lim _{s \rightarrow 1^{-}} f_{1}(s) \in R, \lim _{s \rightarrow 1^{-}} \tilde{f}_{1}(s) \in R,\right.\right.
$$$$
\frac{d}{d s} D_{h}(\widetilde{v}(s)) \sim-c_{h}(1-s)^{\gamma_{h} / p-1}
$$$$
\text { as } s \longrightarrow 1^{-}\left(c_{h}=\text { const. }>0\right) \text {, }
$$$$
\frac{d}{d s} D_{j}(\widetilde{v}(s))=c_{j}(1-s)^{\left(q_{j} \backslash p\right)-1}+\widetilde{f}_{j}(s)
$$

as $j \in\{2, \ldots, n\} \backslash\{h\} \forall s \in\left[0,1\left[\right.\right.$ with $c_{j}=$ const. $>0$,

$$
\begin{gathered}
\lim _{s \rightarrow 1^{-}} \tilde{f}_{j}(s) \in R \quad \text { if } v_{j} \widetilde{v}_{h} \equiv 0, \\
\lim _{s \rightarrow 1^{-}} \frac{d}{d s} D_{j}(\widetilde{v}(s))=-\infty \quad \text { if } v_{j} \widetilde{v}_{h} \neq 0 .
\end{gathered}
$$

Then, $\lim _{s \rightarrow 1^{-}} \sum_{j=1}^{n}(t(\widetilde{v}(s)))^{q_{j}}(d / d s) D_{j}(\widetilde{v}(s))=-\infty$. About (62), let $v \in \mathscr{F}$. Set

$$
\begin{aligned}
& g_{1}(s, \tau)=D_{1}\left(\tau u_{1}^{*}, s v_{2}, \ldots, s v_{n}\right) \\
& =q_{1}^{-1}\left[\int _ { \Omega } \left(d_{1} \tau^{\gamma_{1}}\left(u_{1}^{*}\right)^{\gamma_{1}}\right.\right. \\
& \left.+d s^{\gamma_{1}}\left(\sum_{\ell=2}^{n} v_{\ell}^{\gamma_{1}}\right)\right)^{q_{1} \backslash \gamma_{1}} d x \\
& \left.\quad-\int_{\Omega} \widetilde{d}^{q_{1}}\left(\sum_{\ell=2}^{n} v_{\ell}^{\gamma_{1}}\right)^{q_{1} \backslash \gamma_{1}} d x\right] \quad \forall s, \tau \geq 0 ;
\end{aligned}
$$

$$
\text { as } \varepsilon \longrightarrow 0^{+} \forall s \in[1,+\infty[\text {, }
$$


we have

$$
\begin{aligned}
g_{1}(1,0) & =-1, \\
\frac{\partial g_{1}}{\partial \tau}(s, \tau) & >0 \quad \forall s \geq 0, \quad \forall \tau>0, \\
g_{1}(s, 0) & =-s^{q_{1}}<-1 \quad \forall s>1, \\
\lim _{\tau \rightarrow+\infty} g_{1}(s, \tau) & =+\infty \quad \forall s \geq 0 .
\end{aligned}
$$

Relations (69) imply that

only one $\tau(s)>0$

$$
\begin{aligned}
(\tau(s) & >0 \text { if } s>1, \tau(1)=0) \text { exists such that } \\
g_{1}(s, \tau(s)) & =-1,
\end{aligned}
$$

$\forall s \geq 1$

and the function $\tau(s)$ belongs to $C^{0}\left(\left[1,+\infty[) \cap C^{1}(] 1,+\infty[)\right.\right.$ and it results in

$$
\begin{aligned}
\tau^{\prime}(s)=-\frac{1}{(\tau(s))^{\gamma_{1}-1}} \widetilde{g}_{1}(s, \tau(s)) \\
\left.\forall s>1, \lim _{s \rightarrow 1^{+}} \widetilde{g}_{1}(s, \tau(s)) \in\right]-\infty, 0[.
\end{aligned}
$$

Then, with $\widetilde{v}(s)=\left(\tau(s) u_{1}^{*}, s v_{2}, \ldots, s v_{n}\right)$, we have

$$
\begin{aligned}
& \widetilde{v}(1)=v, \\
& \widetilde{v}(s) \in S\left(D_{1}\right) \quad \forall s \geq 1 ;
\end{aligned}
$$

moreover,

$$
\begin{aligned}
& \left.\lim _{s \rightarrow 1^{+}} \frac{d}{d s} H_{\lambda}(\widetilde{v}(s)) \in\right]-\infty, 0[, \\
& \lim _{s \rightarrow 1^{+}} \frac{d}{d s} D_{j}(\widetilde{v}(s))=+\infty \quad \text { if } v_{j} \neq \equiv 0, \\
& \lim _{s \rightarrow 1^{+}} \frac{d}{d s} D_{j}(\widetilde{v}(s)) \in R \quad \text { if } v_{j} \equiv 0,
\end{aligned}
$$

$$
\text { as } j=2, \ldots, n
$$

from which $\quad \lim _{s \rightarrow 1^{+}}\left[(t(\widetilde{v}(s)))^{p}(d / d s) H_{\lambda}(\widetilde{v}(s)) \quad-\right.$ $\left.\sum_{j=1}^{n}(t(\widetilde{v}(s)))^{q_{j}}(d / d s) D_{j}(\widetilde{v}(s))\right]=-\infty$ since $v_{j} \quad \not \equiv \quad 0$ as some $j \geq 2$.

Finally, let us verify (63), for example, when $h=2$. Let $v \in \mathscr{F}$. Since

$$
\begin{aligned}
& v_{1} \neq \equiv 0 \Longrightarrow \\
& v_{j} \neq 0 \text { as some } j>2,
\end{aligned}
$$

we can suppose $v_{3} \sim 0$. Set $\widetilde{v}(s)=\left(v_{1},(1-\right.$ $\left.s)^{1 / v_{1}} v_{3}, s^{1 / \gamma_{1}} v_{3}, \ldots, v_{n}\right)$; we have

$$
\begin{aligned}
& \widetilde{v}(1)=v, \\
& \widetilde{v}(s) \in S\left(D_{1}\right) \quad \forall s \in[0,1] ;
\end{aligned}
$$

we add that

$$
\begin{aligned}
& \lim _{s \rightarrow 1^{-}} \frac{d}{d s} H_{\lambda}(\widetilde{v}(s)) \in R, \\
& \lim _{s \rightarrow 1^{-}} \frac{d}{d s} D_{2}(\widetilde{v}(s))=-\infty, \\
& \lim _{s \rightarrow 1^{-}} \frac{d}{d s} D_{3}(\widetilde{v}(s))=-\infty, \\
& \left.\lim _{s \rightarrow 1^{-}} \frac{d}{d s} D_{j}(\widetilde{v}(s))<+\infty, \text { as } j>3 \text { (if } n>3\right)
\end{aligned}
$$

from which $\quad \lim _{s \rightarrow 1^{-}}\left[(t(\widetilde{v}(s)))^{p}(d / d s) H_{\lambda}(\widetilde{v}(s))\right.$ $\left.\sum_{j=1}^{n}(t(\widetilde{v}(s)))^{q_{j}}(d / d s) D_{j}(\widetilde{v}(s))\right]=+\infty$.

\section{Neumann Problems}

We assume $W=\left(W^{1, p}(\Omega)\right)^{n}(n \geq 1,1<p<\infty, \Omega \subseteq$ $R^{N}$ is an open, bounded, connected and $C^{0,1}$ set, $\|v\|=$ $\left.\left(\sum_{\ell=1}^{n} \int_{\Omega}\left[\left|\nabla v_{\ell}\right|^{p}+\left|v_{\ell}\right|^{p}\right] d x\right)^{1 / p} \forall v=\left(v_{1}, \ldots, v_{n}\right) \in W\right)$, $B_{\ell}\left(v_{\ell}\right)=p^{-1} \int_{\Omega} b_{\ell}\left|v_{\ell}\right|^{p} d x \forall v_{\ell} \in W^{1, p}(\Omega)$ with $b_{\ell} \in L^{\infty}(\Omega) \backslash$ $\{0\}$, and $\widehat{B}_{\ell}\left(v_{\ell}\right)=p^{-1} \int_{\partial \Omega} \widehat{b}_{\ell}\left|v_{\ell}\right|^{p} d \sigma \forall v_{\ell} \in W^{1, p}(\Omega)$ with $\widehat{b}_{\ell} \in L^{\infty}(\partial \Omega) \backslash\{0\}$ ( $\sigma$ is the measure on $\partial \Omega, v_{\ell}=\gamma_{0}\left(v_{\ell}\right)$, where $\gamma_{0}: W^{1, p}(\Omega) \rightarrow W^{(p-1) / p, p}(\partial \Omega)$ is the trace operator).

Let us consider the functional $A$ (as in $\left(i_{11}\right)$ ) such that $A(v) \geq p^{-1} \tilde{c} \sum_{\ell=1}^{n} \int_{\Omega}\left|\nabla v_{\ell}\right|^{p} d x \forall v \in W(\tilde{c}=$ const. $>0)$. Moreover, $|\cdot|_{N}$ and $\widetilde{p}$ have been settled as in Section 3, $v$ is the outward orthogonal unitary vector to $\partial \Omega, \widehat{p}=(N-1) p /(N-$ $p)$ if $p<N$, and $\widehat{p}=\infty$ if $p \geq N$.

About the validity of assumptions $\left(i_{13}\right)-\left(i_{15}\right)$, we set $C^{*}=$ $\left\{c=\left(c_{1}, \ldots, c_{n}\right) \in R^{n}: c_{\ell}=0\right.$ if $\ell \in I \backslash I^{*}, c_{\ell} \neq 0$ as some $\left.\ell \in I^{*}\right\}\left(I=\{1, \ldots, n\}, I^{*}(\neq \varnothing) \subseteq I\right)$ and we introduce the following assumptions:

$\left(i_{4}\right) I^{*} \subseteq I$ exists: $D_{1}(c)<0$ for every $c \in C^{*}$.

$\left(i_{4}\right) I^{*} \subseteq I$ exists: $D_{1}(c)<0$ and $A(c)=0$ for every $c \in C^{*}$.

Proposition 14. When $b_{\ell} \geq 0$ and $\widehat{b}_{\ell} \geq 0$ as $\ell \in I$, then $\left(i_{13}\right)$ holds if $\lambda_{\ell} \leq 0, \mu_{\ell} \leq 0$, and $\lambda_{\ell}+\mu_{\ell}<0$ as $\ell \in I$. When $\left(i_{4}\right)$ holds with $I^{*} \neq I, b_{\ell} \geq 0$ and $\widehat{b}_{\ell} \geq 0$ as $\ell \in I \backslash I^{*}$, $V^{+}\left(D_{1}\right) \neq \varnothing$, then with $\lambda_{\ell} \leq 0, \mu_{\ell} \leq 0$ and $\lambda_{\ell}+\mu_{\ell}<0$ as $\ell \in I \backslash I^{*} \exists \delta^{*}>0:\left(i_{14}\right)$ holds if $\left|\lambda_{\ell}\right|,\left|\mu_{\ell}\right| \leq \delta^{*} \forall \ell \in I^{*}$. When $\left(i_{4}\right)$ holds with $I^{*}=I$ and $V^{+}\left(D_{1}\right) \neq \varnothing$, then $\exists \delta^{*}>0:\left(i_{14}\right)$ holds if $\left|\lambda_{\ell}\right|,\left|\mu_{\ell}\right| \leq \delta^{*} \forall \ell \in I$.

Proof. We reason by contradiction as in [11, Propositions 3.2 and 3.3].

Proposition 15. When $\left(\underline{i}_{4}\right)$ holds with $I^{*} \neq I, b_{\ell} \geq 0$ and $\widehat{b}_{\ell} \geq$ 0 as $\ell \in I \backslash I^{*}, \int_{\Omega} b_{\ell} d x>0$ and $\int_{\partial \Omega} \widehat{b}_{\ell} d \sigma>0$ as $\ell \in I^{*}$, then with $\lambda_{\ell} \leq 0, \mu_{\ell} \leq 0$, and $\lambda_{\ell}+\mu_{\ell}<0$ as $\ell \in I \backslash I^{*} \exists \delta^{*}>0:\left(i_{15}\right)$ holds if $\lambda_{\ell}, \mu_{\ell} \in\left[0, \delta^{*}\right] \forall \ell \in I^{*}$ and $\lambda_{\ell}+\mu_{\ell}>0$ for some $\ell$. When $\left(i_{4}\right)$ holds with $I^{*}=I, \int_{\Omega} b_{\ell} d x>0$ and $\int_{\partial \Omega} \widehat{b}_{\ell} d \sigma>0$ as $\ell \in I$, then $\exists \delta^{*}>0:\left(i_{15}\right)$ holds if $\lambda_{\ell}, \mu_{\ell} \in\left[0, \delta^{*}\right] \forall \ell \in I$ and $\lambda_{\ell}+\mu_{\ell}>0$ for some $\ell$.

Proof. See [11, Propositions 3.4 and 3.5]. 
Passing to the applications, we state in advance that the results of [8] for problem

$$
\begin{aligned}
-\operatorname{div}\left(|\nabla u|^{p-2} \nabla u\right) & =b(x) u^{s-1} \quad \text { in } \Omega, \\
|\nabla u|^{p-2} \frac{\partial u}{\partial \nu} & =a(x) u^{q-1} \quad \text { on } \partial \Omega
\end{aligned}
$$

can be obtained by using Theorem 1 and Proposition 14, Theorems 2.1 and 2.2 of [10], and Propositions 3.3 and A.4 of [11] (taking into account [17] too).

Application 3. Let $n>1$ and, for each $v=\left(v_{1}, \ldots, v_{n}\right) \in W$. We set

$$
\begin{aligned}
& A(v)=p^{-1} \sum_{\ell=1}^{n} \int_{\Omega}\left|\nabla v_{\ell}\right|^{p} d x, \\
& D_{1}(v)=q_{1}^{-1}\left[\int_{\partial \Omega}\left(\sum_{\ell=1}^{n} d_{\ell}\left|v_{\ell}\right|^{\gamma}\right)^{q_{1} / \gamma} d \sigma\right. \\
& \left.-\sum_{\ell=1}^{n} \int_{\partial \Omega} \widehat{d}_{\ell}\left|v_{\ell}\right|^{q_{1}} d \sigma\right], \\
& D_{2}(v)=-\left.\left.\prod_{\ell=1}^{n}\left|\int_{\partial \Omega} \hat{\rho}_{\ell}\right| v_{\ell}\right|^{\gamma_{\ell}} d \sigma\right|^{\gamma_{\ell}^{\prime} / \gamma_{\ell}},
\end{aligned}
$$

where

$$
\begin{gathered}
1<\gamma<q_{1}<p, \\
1<\gamma_{\ell}<\gamma_{\ell}^{\prime}, \quad \gamma_{\ell}<\widehat{p}, \\
q_{1}<q_{2}=\sum_{\ell=1}^{n} \gamma_{\ell}^{\prime} ; \\
d_{\ell}, \widehat{d}_{\ell} \in L^{\infty}(\partial \Omega), \quad d_{\ell}, \widehat{d}_{\ell}>0 ; \\
\hat{\rho}_{\ell} \in L^{\infty}(\partial \Omega) \backslash\{0\} .
\end{gathered}
$$

Let us consider the system:

$$
\begin{aligned}
& -\operatorname{div}\left(\left|\nabla u_{i}\right|^{p-2} \nabla u_{i}\right)=\lambda_{i} b_{i}\left|u_{i}\right|^{p-2} u_{i} \text { in } \Omega, \\
& \left|\nabla u_{i}\right|^{p-2} \frac{\partial u_{i}}{\partial \nu}=\mu_{i} \widehat{b}_{i}\left|u_{i}\right|^{p-2} u_{i} \\
& +\left(\sum_{\ell=1}^{n} d_{\ell}\left|u_{\ell}\right|^{\gamma}\right)^{q_{1} / \gamma-1} d_{i}\left|u_{i}\right|^{\gamma-2} u_{i}-\widehat{d}_{i}\left|u_{i}\right|^{q_{1}-2} u_{i} \\
& -\left(\left.\left.\prod_{\ell \neq i}\left|\int_{\partial \Omega} \hat{\rho}_{\ell}\right| u_{\ell}\right|^{\gamma_{\ell}} d \sigma\right|^{\gamma_{\ell}^{\prime} / \gamma_{\ell}}\right) \\
& \left.\left.\cdot \gamma_{i}^{\prime}\left|\int_{\partial \Omega} \hat{\rho}_{i}\right| u_{i}\right|^{\gamma_{i}} d \sigma\right|^{\gamma_{\ell}^{\prime} / \gamma_{\ell}-2}\left(\int_{\partial \Omega} \hat{\rho}_{i}\left|u_{i}\right|^{\gamma_{i}} d \sigma\right) \\
& \cdot \hat{\rho}_{i}\left|u_{i}\right|^{\gamma_{i}-2} u_{i} \text { on } \partial \Omega
\end{aligned}
$$

Proposition 16. Let $u=\left(u_{1}, \ldots, u_{n}\right)$ be a weak solution of system (80). Then,

$$
\begin{aligned}
& \lambda_{i} \neq 0, \\
& u_{i} \neq \equiv 0
\end{aligned}
$$

for some $i \in\{1, \ldots, n\} \Longrightarrow u_{i}$ is not constant;

$$
\begin{aligned}
u_{i} \geq 0 \quad \forall i \in\{1, \ldots, n\} \\
\quad \Longrightarrow u_{i} \in L^{\infty}(\Omega) \cap C_{\ell o c}^{1, \alpha_{i}}(\Omega) \quad \forall i \in\{1, \ldots, n\}
\end{aligned}
$$

$u_{i}>0$ if $u_{i} \not \equiv 0$.

Proof. Relation (81) is evident. About (82), it is easy to verify that

$$
\begin{gathered}
\sum_{i=1}^{n} \int_{\Omega}\left|\nabla u_{i}\right|^{p-2} \nabla u_{i} \cdot \nabla v_{i} d x \leq c_{1} \int_{\Omega}\left(\sum_{i=1}^{n} u_{i}+1\right)^{p-1} \\
\cdot\left(\sum_{i=1}^{n} v_{i}\right) d x+\int_{\partial \Omega}\left[c_{2}+c_{3} \sum_{\ell=1}^{n}\left(u_{\ell}+1\right)^{\gamma_{0}-p}\right] \\
\cdot\left(\sum_{i=1}^{n} u_{i}+1\right)^{p-1}\left(\sum_{i=1}^{n} v_{i}\right) d \sigma \\
\forall v=\left(v_{1}, \ldots, v_{n}\right) \in\left(W^{1, p}(\Omega) \cap L^{\infty}(\Omega)\right)^{n} \text { with } v_{i} \geq 0,
\end{gathered}
$$

where $c_{1}-c_{3}$ are positive constants and $\gamma_{0}=\max \left\{\gamma_{1}, \ldots, \gamma_{n}\right\}$. Consequently [11, Proposition A.4], $u_{i} \in L^{\infty}(\Omega)$ from which, since $-\operatorname{div}\left(\left|\nabla u_{i}\right|^{p-2} \nabla u_{i}\right)=\lambda_{i} b_{i}\left|u_{i}\right|^{p-2} u_{i}$ in $\Omega$, we deduce that $u_{i} \in C_{\text {loc }}^{1, \alpha_{i}}(\Omega)$ [17] and $u_{i}>0$ if $u_{i} \not \equiv 0$ [18].

Let us introduce the conditions:

$$
\begin{aligned}
& d_{1}^{q_{1} / \gamma}>\widehat{d}_{1}\left(\Longrightarrow V^{+}\left(D_{1}\right) \neq \varnothing\right), \\
& \left(\sum_{\ell=2}^{n} d_{\ell}\right)^{q_{1} / \gamma}<\min \left\{\widehat{d}_{2}, \ldots, \widehat{d}_{n}\right\} \\
& \left(\Longrightarrow D_{1}\left(0, c_{2}, \ldots, c_{n}\right)<0 \forall\left(c_{2}, \ldots, c_{n}\right) \in R^{n-1} \backslash\{0\}\right), \\
& b_{1} \geq 0, \\
& \widehat{b}_{1} \geq 0, \\
& \int_{\Omega} b_{\ell} d x>0, \\
& \int_{\partial \Omega} \hat{b}_{\ell} d \sigma>0
\end{aligned}
$$

as $\ell=2, \ldots, n$. 
Then (Propositions 14 and 15),

$(84)-(86) \Longrightarrow$

(with $\lambda_{1} \leq 0, \mu_{1} \leq 0, \lambda_{1}+\mu_{1}<0 \exists \delta_{1}^{*}$

$>0:\left(i_{14}\right)$ holds if $\left|\lambda_{\ell}\right|,\left|\mu_{\ell}\right| \leq \delta_{1}^{*} \forall \ell$

$\in\{2, \ldots, n\})$,

$(85)-(87) \Longrightarrow$

(with $\lambda_{1} \leq 0, \mu_{1} \leq 0$ and $\lambda_{1}+\mu_{1}<0 \exists \delta_{2}^{*}$

$>0:\left(i_{15}\right)$ holds if $\lambda_{\ell}, \mu_{\ell} \in\left[0, \delta_{2}^{*}\right] \forall \ell$

$\in\{2, \ldots, n\}$ and $\lambda_{\ell}+\mu_{\ell}>0$ as some $\left.\ell\right)$.

Proposition 17 (Theorems 1 and 4, Remark 6, Proposition 16). Under conditions (79), one has the following:

When (84)-(86) hold, with $\lambda_{\ell}, \mu_{\ell}$ as in (88), system (80) has at least two weak solutions $u^{0}$ and $-u^{0}\left(u^{0}=\right.$ $\tau^{0} v^{0}, \tau^{0}=$ const. $\left.>0, v^{0} \in S_{\lambda \mu} \cap V^{+}\left(D_{1}\right)\right)$, and one has $u_{i}^{0} \in L^{\infty}(\Omega) \cap C_{\ell o c}^{1, \alpha_{i}^{0}}(\Omega), u_{i}^{0} \geq 0$ as $i=1, \ldots, n$, $u_{i}^{0}>0$ if $u_{i}^{0} \not \equiv 0$.

When $q_{2}<p$ and (85)-(87) hold, with $\lambda_{\ell}, \mu_{\ell}$ as in (89), system (80) has at least two weak solutions $\bar{u}$ and $-\bar{u}\left(\bar{u}=\bar{\tau} \bar{v}, \bar{\tau}=\right.$ const. $\left.>0, \bar{v} \in V_{\lambda \mu}^{-} \cap S\left(D_{1}\right)\right)$, and one has $\bar{u}_{i} \in L^{\infty}(\Omega) \cap C_{\ell o c}^{1, \bar{\alpha}_{i}}(\Omega), \bar{u}_{i} \geq 0$ as $i=1, \ldots, n$, $\bar{u}_{i}>0$ if $\bar{u}_{i} \sim 0$.

Consequently, when $q_{2}<p$ and (84)-(87) hold, with $\lambda_{\ell}, \mu_{\ell}$ as in (89) and $\min \left\{\delta_{1}^{*}, \delta_{2}^{*}\right\}$ instead of $\delta_{2}^{*}$, system (80) has at least four different weak solutions.

Proposition 18. One gets the following:

$$
u_{1}^{0} \not \equiv 0 \quad \text { on } \partial \Omega
$$

either $\gamma_{h}^{\prime} \geq p$

$$
\begin{gathered}
\text { or } \gamma \leq \gamma_{h}^{\prime} \Longrightarrow \\
u_{h}^{0} \neq 0
\end{gathered}
$$

as some $h \in\{2, \ldots, n\}$.

Proof. Relation (90) is evident. About (91), it is sufficient (Proposition 2) to verify that

$\left(i_{0}^{h}\right)$ holds with $\mathscr{F}=\left\{v \in S_{\lambda \mu} \cap V^{+}\left(D_{1}\right): v_{\ell} \geq 0\right.$ as $\ell=$ $\left.1, \ldots, n, v_{h} \equiv 0\right\} \neq \varnothing$.

Let us check, for example, the case $h=2$. Let $v \in \mathscr{F}$; then, $\Gamma \subseteq \partial \Omega$ exists such that $\sigma(\Gamma)>0$ and $\sum_{\ell \neq 2} d_{\ell} v_{\ell}^{\gamma}>0$ on $\Gamma$. Let $k \subseteq \Omega$ be a compact set with positive measure and $\Omega^{\prime}$ an open set such that $\bar{\Omega}^{\prime} \subseteq \Omega, k \subseteq \Omega^{\prime}$. Thanks to [11, Propositions A.1,
A.2], a compact set $\widehat{\Gamma} \subseteq \Gamma$ with $\sigma(\widehat{\Gamma})>0,\left(\varphi_{1 \varepsilon}\right)_{0<\varepsilon<\varepsilon_{0}} \subseteq C_{0}^{\infty}(\Omega)$, and $\left(\varphi_{2 \varepsilon}\right)_{0<\varepsilon<\varepsilon_{0}} \subseteq C_{0}^{\infty}\left(R^{N}\right)$ exist such that

$$
0 \leq \varphi_{1 \varepsilon} \leq 1,
$$

$\operatorname{supp} \varphi_{1 \varepsilon} \subseteq \Omega^{\prime}$,

$$
\varphi_{1 \varepsilon} \longrightarrow \chi \text { strongly in } L^{\mathcal{S}}(\Omega)
$$

$\int_{\Omega}\left|\nabla \varphi_{1 \varepsilon}\right|^{s} d x \longrightarrow+\infty \quad$ as $\varepsilon \longrightarrow 0^{+} \forall s \in[1,+\infty[$,

$$
0 \leq \varphi_{2 \varepsilon} \leq 1
$$

$$
\begin{aligned}
& \operatorname{supp} \varphi_{2 \varepsilon} \subseteq R^{N} \backslash \bar{\Omega}^{\prime}, \\
& \varphi_{2 \varepsilon} \longrightarrow \widehat{\chi} \text { strongly in } L^{s}(\partial \Omega), \\
& \int_{R^{N}} \varphi_{2 \varepsilon}^{s} d x \longrightarrow 0 \quad \text { as } \varepsilon \longrightarrow 0^{+} \forall s \in[1,+\infty[
\end{aligned}
$$

where $\chi$ [resp., $\widehat{\chi}]$ is the characteristic function of $K$ [resp., $\widehat{\Gamma}]$. Set $\varphi_{\varepsilon}=\varphi_{1 \varepsilon}+\varphi_{2 \varepsilon}$; let us fix $\varepsilon$ such that

$$
\begin{aligned}
& \delta=p^{-1}\left[\int_{\Omega}\left|\nabla \varphi_{\varepsilon}\right|^{p} d x-\lambda_{2} \int_{\Omega} b_{2} \varphi_{\varepsilon}^{p} d x\right. \\
&\left.-\mu_{2} \int_{\partial \Omega} \widehat{b}_{2} \varphi_{\varepsilon}^{p} d \sigma\right]>0, \\
& \int_{\partial \Omega}\left(\sum_{\ell \neq 2} d_{\ell} v_{\ell}^{\gamma}\right)^{q_{1} / \gamma-1} d_{2} \varphi_{\varepsilon}^{\gamma} d \sigma>0 .
\end{aligned}
$$

Then, with $\widetilde{v}(s)=\left(s^{1 / p} v_{1},(1-s)^{1 / p} \delta^{-1 / p} \varphi_{\varepsilon}, \ldots, s^{1 / p} v_{n}\right)$, we have

$$
\begin{aligned}
& \widetilde{v}(1)=v, \\
& \widetilde{v}(s) \in S_{\lambda \mu} \quad \forall s \in[0,1], \\
& \frac{d}{d s} D_{1}(\widetilde{v}(s)) \sim-\widehat{c}_{1}(1-s)^{(\gamma / p)-1} \\
& \text { as } s \longrightarrow 1^{-}\left(\widehat{c}_{1}=\text { const. }>0\right), \\
& D_{2}(\widetilde{v}(s))=-\widehat{c}_{2} s^{\left(q_{2}-\gamma_{2}^{\prime}\right) / p}(1-s)^{\gamma_{2}^{\prime} / p} \\
& \forall s \in[0,1]\left(\widehat{c}_{2}=\text { const. } \geq 0\right) \\
& \Longrightarrow \lim _{s \rightarrow 1^{-}} \sum_{j=1}^{2}(t(\widetilde{v}(s)))^{q_{j}} \frac{d}{d s} D_{j}(\widetilde{v}(s)) \\
&=-\infty .
\end{aligned}
$$

Proposition 19. Let for some $h \in\{2, \ldots, n\}$ either $\gamma_{h}^{\prime} \geq p$ or $\gamma \leq \gamma_{h}^{\prime}$. Let $b_{h} \geq 0, \lambda_{h}>0$, and $\mu_{h} \int_{\partial \Omega} \widehat{b}_{h} d \sigma \geq 0$. Then, $u_{h}^{0} \not \equiv 0$ on $\partial \Omega$.

Proof. Since in Proposition $18 u_{h}^{0}>0$, it is sufficient (Proposition 2) to prove that 
$\left(i_{0}^{h}\right)$ holds with $\mathscr{F}=\left\{v \in S_{\lambda \mu} \cap V^{+}\left(D_{1}\right): v_{\ell} \geq 0\right.$ as $\ell=$ $1, \ldots, n, v_{h}>0, v_{h} \equiv 0$ on $\left.\partial \Omega\right\} \neq \varnothing$.

Let us suppose, for example, $h=2$. Let $v \in \mathscr{F}$ and $c_{2}=$ const. $>0$. Set $g(s, \tau)=H_{\lambda \mu}\left(s v_{1}, s v_{2}+\tau c_{2}, \ldots\right.$, $\left.s v_{n}\right)=p^{-1}\left[s^{p} \int_{\Omega}\left|\nabla v_{2}\right|^{p} d x-\lambda_{2} \int_{\Omega} b_{2}\left(s v_{2}+\tau c_{2}\right)^{p} d x-\right.$ $\left.\mu_{2} \tau^{p} c_{2}^{p} \int_{\partial \Omega} \widehat{b}_{2} d \sigma\right]+s^{p} H_{\lambda \mu}\left(v_{1}, 0, \ldots, v_{n}\right) \forall s, \tau \geq 0$; we have

$$
\begin{aligned}
g(1,0) & =1, \\
\frac{\partial g}{\partial \tau}(s, \tau) & <0 \quad \forall s \geq 0, \forall \tau>0, \\
g(s, 0) & =s^{p}>1 \quad \forall s>1, \\
\lim _{\tau \rightarrow+\infty} g(s, \tau) & =-\infty \quad \forall s \geq 0 ;
\end{aligned}
$$

then, $\tau:\left[1,+\infty\left[\rightarrow\left[0,+\infty\left[\right.\right.\right.\right.$ belonging to $C^{0}([1,+\infty[) \cap$ $C^{1}(] 1,+\infty[)$ exists such that $g(s, \tau(s))=1 \forall s \geq 1, \tau(s)>0$ if $s>1, \tau(1)=0$, and $\left.\lim _{s \rightarrow 1^{+}} \tau^{\prime}(s) \in\right] 0,+\infty$ [. Consequently, set $\widetilde{v}(s)=\left(s v_{1}, s v_{2}+\tau(s) \mathcal{c}_{2}, \ldots, s v_{n}\right)$; it results in

$$
\begin{aligned}
\widetilde{v}(1) & =v, \\
\widetilde{v}(s) & \in S_{\lambda \mu} \quad \forall s \geq 1, \\
\lim _{s \rightarrow 1^{+}} \frac{d}{d s} D_{1}(\widetilde{v}(s)) & =q_{1} D_{1}(v)>0, \\
\lim _{s \rightarrow 1^{+}} \frac{d}{d s} D_{2}(\widetilde{v}(s)) & =0 \Longrightarrow \\
\lim _{s \rightarrow 1^{+}} \sum_{j=1}^{2}(t(\widetilde{v}(s)))^{q_{j}} \frac{d}{d s} D_{j}(\widetilde{v}(s)) & \in] 0,+\infty[.
\end{aligned}
$$

Proposition 20. If either $\gamma_{1}^{\prime}>\gamma$ or $\int_{\partial \Omega} \widehat{\rho}_{1} d \sigma=0$, it results in

$$
\begin{aligned}
& \bar{u}_{1} \neq \equiv 0 ; \\
& \bar{u}_{1} \neq \equiv \quad \text { on } \partial \Omega \text { when } \lambda_{1}=0 .
\end{aligned}
$$

Proof. About (97) [resp., (98)], it is sufficient (Proposition 5) to prove that

$\left(\underline{1}^{1}\right)$ holds with $\mathscr{F}=\left\{v \in V_{\lambda \mu}^{-} \cap S\left(D_{1}\right): v_{\ell} \geq 0\right.$ as $\ell=$ $1, \ldots, n, v_{1} \equiv 0$ [resp., $v_{1} \equiv 0$ on $\left.\left.\partial \Omega\right]\right\} \neq \varnothing$.

Let $v \in \mathscr{F}$ and $c_{1}=$ const. $>0$. Let $g_{1}(s, \tau)=$ $D_{1}\left(\tau c_{1}, s v_{2}, \ldots, s v_{n}\right)=q_{1}^{-1}\left[\int_{\partial \Omega}\left(d_{1} \tau^{\gamma} c_{1}^{\gamma}+s^{\gamma} \sum_{\ell \neq 1} d_{\ell} v_{\ell}^{\gamma}\right)^{q_{1} / \gamma} d \sigma-\right.$ $\left.\tau^{q_{1}} c_{1}^{q_{1}} \int_{\partial \Omega} \widehat{d}_{1} d \sigma-s^{q_{1}} \sum_{\ell \neq 1} \int_{\partial \Omega} \widehat{d}_{\ell} v_{\ell}^{q_{1}} d \sigma\right] \forall s \geq 0$ and $\forall \tau \geq 0$. We note that

$$
\begin{aligned}
g_{1}(1,0) & =-1, \\
\frac{\partial g_{1}}{\partial \tau}(s, \tau) & >0 \quad \forall s \geq 0, \quad \forall \tau>0, \\
g_{1}(s, 0) & =-s^{q_{1}}<-1 \quad \forall s>1, \\
\lim _{\tau \rightarrow+\infty} g_{1}(s, \tau) & =+\infty \quad \forall s \geq 0 .
\end{aligned}
$$

Then, $\tau:[1,+\infty[\rightarrow[0,+\infty[(\tau(s)>0$ if $s>1, \tau(1)=0)$ belonging to $C^{0}\left(\left[1,+\infty[) \cap C^{1}(] 1,+\infty[)\right.\right.$ exists such that

$$
\begin{aligned}
g_{1}(s, \tau(s)) & =-1 \quad \forall s \geq 1, \\
\tau^{\prime}(s) & =-\frac{1}{(\tau(s))^{\gamma-1}} \widetilde{g}_{1}(s, \tau(s)) \quad \forall s>1,
\end{aligned}
$$

$\left.\lim _{s \rightarrow 1^{+}} \widetilde{g}_{1}(s, \tau(s)) \in\right]-\infty, 0[$,

from which with $\widetilde{v}(s)=\left(\tau(s) \mathcal{c}_{1}, s v_{2}, \ldots, s v_{n}\right)[\operatorname{resp} ., \widetilde{v}(s)=$ $\left.\left(s v_{1}+\tau(s) c_{1}, s v_{2}, \ldots, s v_{n}\right)\right]$ we get

$$
\begin{aligned}
\widetilde{v}(1) & =v, \\
\widetilde{v}(s) & \in S\left(D_{1}\right) \quad \forall s \geq 1, \\
\lim _{s \rightarrow 1^{+}} \frac{d}{d s} D_{2}(\widetilde{v}(s)) & =0, \\
\lim _{s \rightarrow 1^{+}}(t(\widetilde{v}(s)))^{p} \frac{d}{d s} H_{\lambda \mu}(\widetilde{v}(s)) & \in]-\infty, 0[.
\end{aligned}
$$

Evidently, $\bar{u}_{2} \not \equiv 0$ on $\partial \Omega$ if $n=2$.

Proposition 21. When $n>2$ and for some $h \in\{2, \ldots, n\}$ $\int_{\partial \Omega} \widehat{\rho}_{h} d \sigma=0, b_{h} \geq 0, \mu_{h}>0$, then

$$
\bar{u}_{h} \neq \equiv 0 \text { on } \partial \Omega \text {. }
$$

Proof. Let us prove (102) as $h=2$. Reasoning by contradiction, let $\bar{u}_{2} \equiv 0$ on $\partial \Omega$; that is, $\bar{v}_{2} \equiv 0$ on $\partial \Omega$. Let $c_{2}=$ const. $>0$. As $g_{1}(s, \tau)=D_{1}\left(s \bar{v}_{1}, \bar{v}_{2}+\tau c_{2}, \ldots, s \bar{v}_{n}\right)$ $=q_{1}^{-1}\left[\int_{\partial \Omega}\left(d_{2} \tau^{\gamma} c_{2}^{\gamma}+s^{\gamma} \sum_{\ell \neq 2} d_{\ell} \bar{v}_{\ell}^{\gamma}\right)^{q_{1} / \gamma} d \sigma-\tau^{q_{1}} c_{2}^{q_{1}} \int_{\partial \Omega} \widehat{d}_{2} d \sigma-\right.$ $\left.s^{q_{1}} \sum_{\ell \neq 2} \int_{\partial \Omega} \widehat{d}_{\ell} \bar{v}_{\ell}^{q_{1}} d \sigma\right] \forall s, \tau \geq 0$, we have

$$
\begin{aligned}
g_{1}(s, 0) & \left.=-s^{q_{1}}>-1 \quad \forall s \in\right] 0,1[, \\
\lim _{\tau \rightarrow+\infty} g_{1}(s, \tau) & =-\infty \quad \forall s \geq 0 .
\end{aligned}
$$

Thanks to (103), (104), for each $s \in] 0,1$ [, it is possible to choose $\tau(s)>0$ such that $g_{1}(s, \tau(s))=-1$. Then, set $\widetilde{v}(s)=$ $\left(s \bar{v}_{1}, \bar{v}_{2}+\tau(s) \mathcal{c}_{2}, \ldots, s \bar{v}_{n}\right)$; it results in

$$
\left.D_{1}(\widetilde{v}(s))=-1 \quad \forall s \in\right] 0,1[;
$$

moreover, since

$$
\begin{aligned}
& H_{\lambda \mu}(\widetilde{v}(s))=p^{-1}\left[\int_{\Omega}\left|\nabla \bar{v}_{2}\right|^{p} d x\right. \\
& -\lambda_{2} \int_{\Omega} b_{2}\left(\bar{v}_{2}+\tau(s) c_{2}\right)^{p} d x \\
& \left.-\mu_{2}(\tau(s))^{p} c_{2}^{p} \int_{\partial \Omega} \widehat{b}_{2} d \sigma\right]+s^{p} H_{\lambda \mu}\left(\bar{v}_{1}, 0, \ldots, \bar{v}_{n}\right) \\
& \quad<p^{-1}\left[\int_{\Omega}\left|\nabla \bar{v}_{2}\right|^{p} d x-\lambda_{2} \int_{\Omega} b_{2} \bar{v}_{2}^{p} d x\right] \\
& +s^{p} H_{\lambda \mu}\left(\bar{v}_{1}, 0, \ldots, \bar{v}_{n}\right) \longrightarrow H_{\lambda \mu}(\bar{v})<0 \\
& \text { as } s \longrightarrow 1^{-},
\end{aligned}
$$


$\bar{s} \in] 0,1[$ exists such that

$$
\left.H_{\lambda \mu}(\widetilde{v}(s))<0 \quad \forall s \in\right] \bar{s}, 1[.
$$

Relations (105), (107) imply that (Theorem 4)

$$
\widetilde{\widetilde{E}}(\widetilde{v}(s)) \geq \widetilde{\widetilde{E}}(\bar{v}) \quad \forall s \in] \bar{s}, 1[
$$

Since $D_{2}(\bar{v})=D_{2}(\widetilde{v}(s))=0$, we have

$$
\begin{aligned}
p(t(\bar{v}))^{p-1} H_{\lambda \mu}(\bar{v})+q_{1}(t(\bar{v}))^{q_{1}-1} & =0, \\
p(t(\widetilde{v}(s)))^{p-1} H_{\lambda \mu}(\widetilde{v}(s))+q_{1}(t(\widetilde{v}(s)))^{q_{1}-1} & =0
\end{aligned}
$$$$
\forall s \in] 0,1[,
$$

from which

$$
\begin{aligned}
\widetilde{\widetilde{E}}(\bar{v}) & =\delta\left|H_{\lambda \mu}(\bar{v})\right|^{-q_{1} /\left(p-q_{1}\right)}, \\
\widetilde{\widetilde{E}}(\widetilde{v}(s)) & \left.=\delta\left|H_{\lambda \mu}(\widetilde{v}(s))\right|^{-q_{1} /\left(p-q_{1}\right)} \quad \forall s \in\right] 0,1[,
\end{aligned}
$$

where $\delta=\left(q_{1} / p\right)^{q_{1} /\left(p-q_{1}\right)}-\left(q_{1} / p\right)^{p /\left(p-q_{1}\right)}>0$.

From (108), (110), we get

$$
\left.H_{\lambda \mu}(\bar{v}) \leq H_{\lambda \mu}(\widetilde{v}(s)) \quad \forall s \in\right] \bar{s}, 1[.
$$

We add that since

$$
\begin{aligned}
& \frac{\partial g_{1}}{\partial \tau}(s, \tau)=\tau^{\gamma-1} \widetilde{g}(s, \tau) \quad \forall s, \tau \geq 0, \\
&\left.\lim _{\substack{s \rightarrow 1^{-} \\
\tau \rightarrow 0^{+}}} \widetilde{g}(s, \tau) \in\right] 0,+\infty[
\end{aligned}
$$

then $\left.s_{0} \in\right] \bar{s}, 1\left[\right.$ and $\left.\tau_{0} \in\right] 0,1[$ exist such that

$$
\left.\frac{\partial g_{1}}{\partial \tau}(s, \tau)>0 \quad \forall s \in\right] s_{0}, 1[, \forall \tau \in] 0, \tau_{0}[
$$

from which

$$
\left.\tau(s) \geq \tau_{0} \quad \forall s \in\right] s_{0}, 1[
$$

thanks to (103). Relations (111), (114) imply that

$$
\begin{aligned}
H_{\lambda \mu}(\bar{v}) \leq & -p^{-1}(\tau(s))^{p} c_{2}^{p} \mu_{2} \int_{\partial \Omega} \widehat{b}_{2} d \sigma \\
& +p^{-1}\left[\int_{\Omega}\left|\nabla \bar{v}_{2}\right|^{p} d x-\lambda_{2} \int_{\Omega} b_{2} \bar{v}_{2}^{p} d x\right] \\
& +s^{p} H_{\lambda \mu}\left(\bar{v}_{1}, 0, \ldots, \bar{v}_{n}\right) \\
\leq & -p^{-1} \tau_{0}^{p} c_{2}^{p} \mu_{2} \int_{\partial \Omega} \widehat{b}_{2} d \sigma \\
& +p^{-1}\left[\int_{\Omega}\left|\nabla \bar{v}_{2}\right|^{p} d x-\lambda_{2} \int_{\Omega} b_{2} \bar{v}_{2}^{p} d x\right] \\
& \left.+s^{p} H_{\lambda \mu}\left(\bar{v}_{1}, 0, \ldots, \bar{v}_{n}\right) \quad \forall s \in\right] s_{0}, 1[
\end{aligned}
$$

from which, as $s \rightarrow 1^{-}$, we get the contradiction

$$
\begin{aligned}
H_{\lambda \mu}(\bar{v}) & \leq-p^{-1} \tau_{0}^{p} c_{2}^{p} \mu_{2} \int_{\partial \Omega} \widehat{b}_{2} d \sigma+H_{\lambda \mu}(\bar{v}) \\
& <H_{\lambda \mu}(\bar{v})
\end{aligned}
$$

Application 4. Let $n>1$ and, for each $v=\left(v_{1}, \ldots, v_{n}\right) \in W$, we set

$$
\begin{aligned}
& A(v)=p^{-1} \int_{\Omega}\left(\sum_{\ell=1}^{n}\left|\nabla v_{\ell}\right|^{\gamma}\right)^{p / \gamma} d x, \\
& D_{1}(v)=q_{1}^{-1} \int_{\Omega} \rho_{1}\left(\sum_{\ell=1}^{n} d_{\ell}\left|v_{\ell}\right|^{\gamma_{1}}\right)^{q_{1} / \gamma_{1}} d x, \\
& D_{j}(v)=q_{j}^{-1}\left[\int_{\Omega}\left(\sum_{\ell=1}^{n} d_{j \ell}\left|v_{\ell}\right|^{\gamma_{j}}\right)^{q_{j} / \gamma_{j}} d x\right. \\
& \left.-\sum_{\ell=1}^{n} \int_{\Omega} \tilde{d}_{j \ell}\left|v_{\ell}\right|^{q_{j}} d x\right] \text { as } j=2, \ldots, m,
\end{aligned}
$$

where

$$
\begin{aligned}
& 1<\gamma<p, \\
& 1<\gamma_{j}<q_{j} \text { as } j=1, \ldots, m \text {, } \\
& q_{1}<p, \\
& q_{1}<q_{2}<\cdots<q_{m}<\tilde{p} ; \\
& \rho_{1} \in C^{0}(\Omega) \cap L^{\infty}(\Omega), \\
& d_{\ell}=\text { const. }>0 \text {; } \\
& d_{j \ell}, \tilde{d}_{j \ell} \in L^{\infty}(\Omega) \text {, } \\
& d_{j \ell}, \tilde{d}_{j \ell}>0 \text {, } \\
& \left(\sum_{\ell=1}^{n} d_{j \ell}\right)^{q_{j} / \gamma_{j}} \leq \min \left\{\tilde{d}_{j 1}, \ldots, \tilde{d}_{j n}\right\} \\
& -\operatorname{div}\left[\left(\sum_{\ell=1}^{n}\left|\nabla u_{\ell}\right|^{\gamma}\right)^{p / \gamma-1}\left|\nabla u_{i}\right|^{\gamma-2} \nabla u_{i}\right]=\lambda_{i} b_{i}\left|u_{i}\right|^{p-2} u_{i} \\
& +\rho_{1}\left(\sum_{\ell=1}^{n} d_{\ell}\left|u_{\ell}\right|^{\gamma_{1}}\right)^{q_{1} / \gamma_{1}-1} d_{i}\left|u_{i}\right|^{\gamma_{1}-2} u_{i} \\
& +\sum_{j=2}^{m}\left[\left(\sum_{\ell=1}^{n} d_{j \ell}\left|u_{\ell}\right|^{\gamma_{j}}\right)^{q_{j} / \gamma_{j}-1} d_{j i}\left|u_{i}\right|^{\gamma_{j}-2} u_{i}\right.
\end{aligned}
$$




$$
\begin{array}{r}
\left.-\widetilde{d}_{j i}\left|u_{i}\right|^{q_{j}-2} u_{i}\right] \quad \text { in } \Omega, \\
\left(\sum_{\ell=1}^{n}\left|\nabla u_{\ell}\right|^{\gamma}\right)^{(p / \gamma)-1}\left|\nabla u_{i}\right|^{\gamma-2} \frac{\partial u_{i}}{\partial \nu}=\mu_{i} \widehat{b}_{i}\left|u_{i}\right|^{p-2} u_{i} \\
\quad \text { on } \partial \Omega \text { as } i=1, \ldots, n .
\end{array}
$$

Proposition 22. Let either $q_{m}<\widehat{p}$ or $\mu_{\ell}=0 \forall \ell \in\{1, \ldots, n\}$. If $u=\left(u_{1}, \ldots, u_{n}\right)$ is a weak solution of system (119) with all nonnegative components, then $u_{i} \in L^{\infty}(\Omega) \forall i \in\{1, \ldots, n\}$.

Proof. The statement is true in virtue of [11, Proposition A.4, Remark A.5], and of relation

$$
\begin{aligned}
& \sum_{i=1}^{n} \int_{\Omega}\left(\sum_{\ell=1}^{n}\left|\nabla u_{\ell}\right|^{\gamma}\right)^{(p / \gamma)-1}\left|\nabla u_{i}\right|^{\gamma-2} \nabla u_{i} \cdot \nabla v_{i} d x \\
& \quad \leq \int_{\Omega}\left[c_{1}+c_{2}\left(\sum_{\ell=1}^{n} u_{\ell}+1\right)^{q_{m}-p}\right]\left(\sum_{i=1}^{n} u_{i}+1\right)^{p-1} \\
& \cdot\left(\sum_{i=1}^{n} v_{i}\right) d x+c_{3} \max _{\ell}\left|\mu_{\ell}\right| \int_{\partial \Omega}\left(\sum_{i=1}^{n} u_{i}+1\right)^{p-1} \\
& \cdot\left(\sum_{i=1}^{n} v_{i}\right) d \sigma \\
& \forall v=\left(v_{1}, \ldots, v_{n}\right) \in\left(W^{1, p}(\Omega) \cap L^{\infty}(\Omega)\right)^{n} \text { with } v_{i} \geq 0
\end{aligned}
$$

where $c_{1}-c_{3}$ are positive constants.

Let us introduce the conditions:

$$
\begin{aligned}
& \rho_{1}^{+} \neq 0\left(\Longrightarrow V^{+}\left(D_{1}\right) \neq \varnothing\right), \\
& \int_{\Omega} \rho_{1} d x<0\left(\Longrightarrow D_{1}(c)<0 \forall c \in R^{n} \backslash\{0\}\right), \\
& \int_{\Omega} b_{\ell} d x>0, \\
& \int_{\partial \Omega} \widehat{b}_{\ell} d \sigma>0
\end{aligned}
$$

$$
\text { as } \ell=1, \ldots, n \text {. }
$$

We have (Propositions 14 and 15)

$$
\begin{aligned}
& (121),(122) \Longrightarrow \\
& \left(\exists \delta_{1}^{*}>0:\left(i_{14}\right) \text { holds if }\left|\lambda_{\ell}\right|,\left|\mu_{\ell}\right| \leq \delta_{1}^{*} \forall \ell\right. \\
& \quad \in\{1, \ldots, n\}), \\
& (122),(123) \Longrightarrow \\
& \left(\exists \delta_{2}^{*}>0:\left(i_{15}\right) \text { holds if } \lambda_{\ell}, \mu_{\ell} \in\left[0, \delta_{2}^{*}\right] \forall \ell\right. \\
& \left.\quad \in\{1, \ldots, n\}, \lambda_{\ell}+\mu_{\ell}>0 \text { as some } \ell\right) .
\end{aligned}
$$

Proposition 23 (see Theorems 1 and 4, Remark 6). Under conditions (118), one has the following:

When (121) and (122) hold, with $\lambda_{\ell}, \mu_{\ell}$ as in (124), system (119) has at least two weak solutions $u^{0}$ and $-u^{0}\left(u^{0}=\tau^{0} v^{0}, \tau^{0}=\right.$ const. $\left.>0, v^{0} \in S_{\lambda \mu} \cap V^{+}\left(D_{1}\right)\right)$, and one has $u_{i}^{0} \geq 0$ as $i=1, \ldots, n$.

When $q_{m}<p$ and (122), (123) hold, with $\lambda_{\ell}, \mu_{\ell}$ as in (125), system (119) has at least two weak solutions $\bar{u}$ and $-\bar{u}\left(\bar{u}=\bar{\tau} v, \bar{\tau}=\right.$ const. $\left.>0, \bar{v} \in V_{\lambda \mu}^{-} \cap S\left(D_{1}\right)\right)$, and one has $\bar{u}_{i} \geq 0$ as $i=1, \ldots, n$.

Consequently, when $q_{m}<p$ and (121)-(123) hold, with $\lambda_{\ell}, \mu_{\ell}$ as in (125) and $\min \left\{\delta_{1}^{*}, \delta_{2}^{*}\right\}$ instead of $\delta_{2}^{*}$, system (119) has at least four different weak solutions.

From Propositions 22 and 23, we deduce the following.

Proposition 24. We have $\bar{u}_{i} \in L^{\infty}(\Omega)$ as $i=1, \ldots, n$. If either $q_{m}<\widehat{p}$ or $\mu_{\ell}=0 \forall \ell \in\{1, \ldots, n\}$, then $u_{i}^{0} \in L^{\infty}(\Omega)$ as $i=$ $1, \ldots, n$.

Proposition 25. Let $\Omega_{0}=\left\{x \in \Omega: \rho_{1}(x)=0\right\}$. If $\gamma>\gamma_{1}$ and $\left|\Omega_{0}\right|_{N}=0$, then $u_{i}^{0} \not \equiv 0$ as $i=1, \ldots, n$.

Proof. It is sufficient (Proposition 2) to show that

$$
\begin{aligned}
& \text { as } h=1, \ldots, n\left(i_{0}^{h}\right) \text { holds with } \mathscr{F}=\left\{v \in S_{\lambda \mu} \cap\right. \\
& \left.V^{+}\left(D_{1}\right): v_{\ell} \geq 0 \text { as } \ell=1, \ldots, n, v_{h} \equiv 0\right\} \neq \varnothing .
\end{aligned}
$$

Let us consider, for example, the case $h=1$. Let $v \in \mathscr{F}$. Firstly, let us prove that $\widetilde{v}_{1} \in W^{1, p}(\Omega)$ exists with $\widetilde{v}_{1} \geq 0$ such that

$$
\begin{aligned}
& \delta=\left[\int_{\Omega}\left|\nabla \widetilde{v}_{1}\right|^{p} d x-\lambda_{1} \int_{\Omega} b_{1} \widetilde{v}_{1}^{p} d x-\mu_{1} \int_{\partial \Omega} \widehat{b}_{1} \tilde{v}_{1}^{p} d \sigma\right] \\
& >0 \\
& \int_{\Omega}\left(\sum_{\ell \neq 1}\left|\nabla v_{\ell}\right|^{\gamma}\right)^{(p / \gamma)-1}\left|\nabla \widetilde{v}_{1}\right|^{\gamma} d x>0, \\
& \int_{\Omega} \rho_{1}\left(\sum_{\ell \neq 1} d_{\ell} v_{\ell}^{\gamma_{1}}\right)^{q_{1} / \gamma_{1}-1} \widetilde{v}_{1}^{\gamma_{1}} d x>0 .
\end{aligned}
$$

Let $\Omega^{+}=\left\{x \in \Omega: \rho_{1}(x)>0\right\}$ and $\Omega_{\ell}=\left\{x \in \Omega:\left|\nabla v_{\ell}(x)\right|>\right.$ $0\}(\ell=1, \ldots, n)$. Since $v \in V^{+}\left(D_{1}\right)$, then $\ell_{0} \in\{2, \ldots, n\}$ exists such that $\left|\Omega_{\ell_{0}}\right|_{N}>0$. In fact, $\left|\Omega_{\ell}\right|_{N}=0 \forall \ell \in\{2, \ldots, n\} \Rightarrow$ $v_{\ell}=$ const. $\forall \ell \in\{2, \ldots, n\} \Rightarrow D_{1}(v)<0$.

Firstly, let us suppose $\left|\Omega_{\ell_{0}} \cap \Omega^{+}\right|_{N}>0$. Then, a compact set $K_{0} \subseteq \Omega^{+}$exists such that $\left|K_{0}\right|_{N}>0$ and $\left|\nabla v_{\ell_{0}}\right|>0$ in $K_{0}$. Let [11, Proposition A.1] $\left(\varphi_{\varepsilon}\right)_{0<\varepsilon<\varepsilon_{0}} \subseteq C_{0}^{\infty}\left(\Omega^{+}\right)$with $0 \leq \varphi_{\varepsilon} \leq 1$ such that

$$
\varphi_{\varepsilon} \longrightarrow \chi \quad \text { strongly in } L^{s}(\Omega)
$$

$$
\int_{\Omega}\left|\nabla \varphi_{\varepsilon}\right|^{s} d x \longrightarrow+\infty
$$


where $\chi$ is the characteristic function of $K_{0}$. Set $\Omega_{\varepsilon}=\{x \in$ $\left.\Omega_{\ell_{0}}:\left|\nabla \varphi_{\varepsilon}(x)\right|>0\right\}$ and $J=\{\varepsilon \in] 0, \varepsilon_{0}\left[:\left|\Omega_{\varepsilon}\right|_{N}>0\right\}$; we can consider two cases:

$$
\begin{array}{r}
\inf J=0, \\
\text { either } J=\varnothing \\
\text { or inf } J>0 .
\end{array}
$$

In case (130), thanks to (129), it is possible to choose $\varepsilon$ such that (126)-(128) hold with $\widetilde{v}_{1}=\varphi_{\varepsilon}$. In case (131), also from (129), we can find $\varepsilon$ such that $\widetilde{v}_{1}=\varphi v_{\ell_{0}}+\varphi_{\varepsilon}$ satisfying (126)(128), where $\varphi \in C_{0}^{\infty}\left(\Omega^{+}\right), 0 \leq \varphi \leq 1$, and $\varphi=1$ in $K_{0}$. have

Let us suppose now $\left|\Omega_{\ell_{0}} \cap \Omega^{+}\right|_{N}=0$. Since $\left|\Omega_{0}\right|_{N}=0$, we

$$
\begin{gathered}
\exists \text { a compact set } K_{0} \subseteq \Omega \backslash \bar{\Omega}^{+}: \\
\left|K_{0}\right|_{N}>0, \\
\left|\nabla v_{\ell_{0}}\right|>0
\end{gathered}
$$

in $K_{0}$.

Let us add that

$$
\begin{aligned}
& v \in V^{+}\left(D_{1}\right) \Longrightarrow \\
& \exists \ell_{1} \in\{2, \ldots, n\}, \\
& \text { a compact set } K_{1} \subseteq \Omega^{+}: \\
&\left|K_{1}\right|_{N}>0, \\
& v_{\ell_{1}}>0
\end{aligned}
$$

in $K_{1}$.

Let $\left(\varphi_{\varepsilon}\right)_{0<\varepsilon<\varepsilon_{0}} \subseteq C_{0}^{\infty}\left(\Omega^{+}\right)$such that $0 \leq \varphi_{\varepsilon} \leq 1, \varphi_{\varepsilon} \rightarrow$ $\chi$ strongly in $L^{s}(\Omega)$ and $\int_{\Omega}\left|\nabla \varphi_{\varepsilon}\right|^{s} d x \rightarrow+\infty$ as $\varepsilon \rightarrow$ $0^{+} \forall s \in\left[1,+\infty\right.$ [, where $\chi$ is the characteristic function of $K_{1}$. Evidently, $\widetilde{v}_{1}=\eta \varphi v_{\ell_{0}}+\varphi_{\varepsilon}$ satisfies (126)-(128) with suitable $\eta>0$ and $\varepsilon, \varphi \in C_{0}^{\infty}\left(\Omega \backslash \bar{\Omega}^{+}\right), 0 \leq \varphi \leq 1$, and $\varphi=1$ in $K_{0}$.

Let us introduce the function $g(s, \tau)=H_{\lambda \mu}\left(\tau \widetilde{v}_{1}, s v_{2}\right.$, $\left.\ldots, s v_{n}\right)=p^{-1}\left[\int_{\Omega}\left(\tau^{\gamma}\left|\nabla \widetilde{v}_{1}\right|^{\gamma}+s^{\gamma} \sum_{\ell \neq 1}\left|\nabla v_{\ell}\right|^{\gamma}\right)^{p / \gamma} d x-\right.$ $\tau^{p}\left(\lambda_{1} \int_{\Omega} b_{1} \widetilde{v}_{1}^{p} d x+\mu_{1} \int_{\partial \Omega} \widehat{b}_{1} \widetilde{v}_{1}^{p} d \sigma\right)-s^{p} \sum_{\ell \neq 1}\left(\lambda_{\ell} \int_{\Omega} v_{\ell}^{p} d x+\right.$ $\left.\left.\mu_{\ell} \int_{\partial \Omega} \widehat{b}_{\ell} v_{\ell}^{p} d \sigma\right)\right] \forall s, \tau \geq 0$. Equation (126) implies that

$$
\begin{aligned}
\lim _{\tau \rightarrow+\infty} g(s, \tau) & =+\infty \quad \forall s \geq 0 \\
& \frac{\partial g}{\partial \tau}(s, \tau) \geq \delta \tau^{p-1}>0 \quad \forall s \geq 0, \forall \tau>0 .
\end{aligned}
$$

Consequently, since $g(1,0)=1$ and $g(s, 0)=s^{p}<1 \forall s \in$ ] $0,1[, \exists \tau:] 0,1] \rightarrow[0,+\infty[(\tau(1)=0$ and $\tau(s)>0$ if $s<$ 1) belonging to $\left.\left.C^{0}(] 0,1\right]\right) \cap C^{1}(] 0,1[)$ such that $g(s, \tau(s))=$ $1 \forall s \in] 0,1]$, and we have

$$
\left.\tau^{\prime}(s)=-\frac{1}{(\tau(s))^{\gamma-1}} \widetilde{g}(s, \tau(s)) \quad \forall s \in\right] 0,1[;
$$

and then, $\left.\lim _{s \rightarrow 1^{-}} \tilde{g}(s, \tau(s)) \in\right] 0,+\infty[$ by $(127)$.
In conclusion, set $\widetilde{v}(s)=\left(\tau(s) \widetilde{v}_{1}, s v_{2}, \ldots, s v_{n}\right)$ and, taking into account (128), (135), it results in

$$
\begin{aligned}
& \widetilde{v}(1)=v, \\
& \left.\left.\widetilde{v}(s) \in S_{\lambda \mu} \quad \forall s \in\right] 0,1\right], \\
& \lim _{s \rightarrow 1^{-}} \frac{d}{d s} D_{1}(\widetilde{v}(s))=-\infty, \\
& \lim _{s \rightarrow 1^{-}} \frac{d}{d s} D_{j}(\widetilde{v}(s))<+\infty \quad \text { as } j \neq 1 \\
& \Longrightarrow \lim _{s \rightarrow 1^{-}} \sum_{j=1}^{m}(t(\widetilde{v}(s)))^{q_{j}} \frac{d}{d s} D_{j}(\widetilde{v}(s))=-\infty .
\end{aligned}
$$

Proposition 26. If for some $j_{0} \in\{2, \ldots, m\} \gamma_{j_{0}}<\gamma \leq \gamma_{1}$, then $u_{i}^{0} \not \equiv 0$ as $i=1, \ldots, n$.

Proof. It is sufficient (Proposition 2) to show that

$$
\begin{aligned}
& \text { as } h=1, \ldots, n\left(i_{0}^{h}\right) \text { holds with } \mathscr{F}=\left\{v \in S_{\lambda \mu} \cap\right. \\
& \left.V^{+}\left(D_{1}\right): v_{\ell} \geq 0 \text { as } \ell=1, \ldots, n, v_{h} \equiv 0\right\} \neq \varnothing .
\end{aligned}
$$

Let us suppose $h=1$ and let $v \in \mathscr{F}$. Let us verify that $\widetilde{v}_{1} \in$ $W^{1, p}(\Omega)$ exists with $\widetilde{v}_{1} \geq 0$ such that (126) and (127) hold. At this aim, we note that

$$
\begin{aligned}
& v \in V^{+}\left(D_{1}\right) \Longrightarrow \\
& \exists \ell_{0} \in\{2, \ldots, n\}: \\
&\left|\Omega_{\ell_{0}}\right|_{N}>0,
\end{aligned}
$$

where $\Omega_{\ell_{0}}=\left\{x \in \Omega:\left|\nabla v_{\ell_{0}}(x)\right|>0\right\}$. Let $K \subseteq \Omega$ be a compact set with positive measure. Let $\left(\varphi_{\varepsilon}\right)_{0<\varepsilon<\varepsilon_{0}} \subseteq C_{0}^{\infty}(\Omega)$ such that $0 \leq \varphi_{\varepsilon} \leq 1, \varphi_{\varepsilon} \rightarrow \chi$ strongly in $L^{s}(\Omega)$ and $\int_{\Omega}\left|\nabla \varphi_{\varepsilon}\right|^{s} d x \rightarrow$ $+\infty$ as $\varepsilon \rightarrow 0^{+} \forall s \in[1,+\infty[(\chi=$ characteristic function of $K)$. With $\Omega_{\varepsilon}$ and $J$ as in Proposition 25, it is possible to find $\varepsilon$ such that $\widetilde{v}_{1}=\varphi_{\varepsilon}$ [resp., $\widetilde{v}_{1}=v_{\ell_{0}}+\varphi_{\varepsilon}$ ] satisfying (126) and (127) in case (130) [resp., (131)]. Then, if $\tau(s)$ and $\widetilde{v}(s)$ are as in Proposition 25, we have

$$
\begin{aligned}
& \widetilde{v}(1)=v, \\
& \left.\left.\widetilde{v}(s) \in S_{\lambda \mu} \quad \forall s \in\right] 0,1\right], \\
& \lim _{s \rightarrow 1^{-}} \frac{d}{d s} D_{j_{0}}(\widetilde{v}(s))=-\infty, \\
& \lim _{s \rightarrow 1^{-}} \frac{d}{d s} D_{j}(\widetilde{v}(s))<+\infty \quad \text { as } j \neq j_{0} \\
& \Longrightarrow \lim _{s \rightarrow 1^{-}} \sum_{j=1}^{m}(t(\widetilde{v}(s)))^{q_{j}} \frac{d}{d s} D_{j}(\widetilde{v}(s))=-\infty .
\end{aligned}
$$

Proposition 27. If for some $j_{0} \in\{2, \ldots, m\} \gamma_{j_{0}}<\gamma_{1} \leq \gamma$, then $\bar{u}_{i} \not \equiv 0$ as $i=1, \ldots, n$. 
Proof. It is sufficient (Proposition 5) to show that

$$
\begin{aligned}
& \text { as } h=1, \ldots, n\left(\underline{i}^{h}\right) \text { holds with } \mathscr{F}=\left\{v \in V_{\lambda \mu}^{-} \cap S\left(D_{1}\right):\right. \\
& \left.v_{\ell} \geq 0 \text { as } \ell=1, \ldots, n, v_{h} \equiv 0\right\}, \text { if } \mathscr{F} \neq \varnothing .
\end{aligned}
$$

Let us suppose, for example, $h=1$ and let us set $\Omega^{-}=$ $\left\{x \in \Omega: \rho_{1}(x)<0\right\}$. Let $K \subseteq \Omega^{-}$be a compact set such that $|K|_{N}>0$ and $v_{\ell_{0}}>0$ in $K$ for some $\ell_{0} \in\{2, \ldots, n\}$. Let $\varphi \in C_{0}^{\infty}\left(\Omega^{-}\right)$with $0 \leq \varphi \leq 1$ and $\varphi=1$ in $K$. Set $g_{1}(s, \tau)=D_{1}\left(\tau \varphi, s v_{2}, \ldots, s v_{n}\right)=q_{1}^{-1} \int_{\Omega} \rho_{1}\left(d_{1} \tau^{\gamma_{1}} \varphi^{\gamma_{1}}+\right.$ $\left.s^{\gamma_{1}} \sum_{\ell \neq 1} d_{\ell} v_{\ell}^{\gamma_{1}}\right)^{q_{1} / \gamma_{1}} d x \forall s, \tau \geq 0$; it results in

$$
\begin{aligned}
g_{1}(1,0) & =-1, \\
\frac{\partial g_{1}}{\partial \tau}(s, \tau) & <0 \quad \forall s \geq 0, \forall \tau>0, \\
g_{1}(s, 0) & \left.=-s^{q_{1}}>-1 \quad \forall s \in\right] 0,1[, \\
\lim _{\tau \rightarrow+\infty} g_{1}(s, \tau) & =-\infty \quad \forall s \geq 0 .
\end{aligned}
$$

Then, $\exists \tau:] 0,1] \rightarrow[0,+\infty[(\tau(1)=0, \tau(s)>0$ if $s<$ 1) belonging to $\left.\left.C^{0}(] 0,1\right]\right) \cap C^{1}(] 0,1[)$ such that $g_{1}(s, \tau(s))=$ $-1 \forall s \in] 0,1]$, and we have

$$
\begin{aligned}
\tau^{\prime}(s)=-\frac{1}{(\tau(s))^{\gamma_{1}-1}} \tilde{g}_{1}(s, \tau(s)) \\
\quad \forall s \in] 0,1\left[, \lim _{s \rightarrow 1^{-}} \widetilde{g}_{1}(s, \tau(s)) \in\right] 0,+\infty[
\end{aligned}
$$

Consequently, with $\widetilde{v}(s)=\left(\tau(s) \varphi, s v_{2}, \ldots, s v_{n}\right)$, we get

$$
\begin{aligned}
& \widetilde{v}(1)=v, \\
& \left.\left.\widetilde{v}(s) \in S\left(D_{1}\right) \quad \forall s \in\right] 0,1\right], \\
& \left.\lim _{s \rightarrow 1^{-}} \frac{d}{d s} H_{\lambda \mu}(\widetilde{v}(s)) \in\right]-\infty, 0[, \\
& \lim _{s \rightarrow 1^{-}} \frac{d}{d s} D_{j_{0}}(\widetilde{v}(s))=-\infty, \\
& \lim _{s \rightarrow 1^{-}} \frac{d}{d s} D_{j}(\widetilde{v}(s))<+\infty \text { as } j \neq j_{0} \\
& \Longrightarrow \lim _{s \rightarrow 1^{-}}\left[(t(\widetilde{v}(s)))^{p} \frac{d}{d s} H_{\lambda \mu}(\widetilde{v}(s))\right. \\
& \left.-\sum_{j=1}^{m}(t(\widetilde{v}(s)))^{q_{j}} \frac{d}{d s} D_{j}(\widetilde{v}(s))\right]=+\infty .
\end{aligned}
$$

\section{Conflict of Interests}

The authors declare that there is no conflict of interests regarding the publication of this paper.

\section{References}

[1] P. Drabek and S. I. Pohozaev, "Positive solutions for the $p$ Laplacian: application of the fibering method," Proceedings of the Royal Society of Edinburgh Section A, vol. 127, no. 4, pp. 703726, 1997.

[2] D. G. Figueiredo, P. L. Lions, and R. Nussbaum, "Positive solutions of a semilinear elliptic problem," Journal de Mathématiques Pures et Appliquées, vol. 61, pp. 41-63, 1982.

[3] P. Clément, D. G. de Figueiredo, and E. Mitidieri, "Positive splutions of semilinear elliptic systems," Communications in Partial Differential Equations, vol. 17, no. 5-6, pp. 923-940, 1992.

[4] D. G. Figueiredo and R. Chiappinelli, "Bifurcation from infinity and multiple solutions for an elliptic system," Differential and Integral Equations, vol. 6, no. 4, pp. 757-771, 1993.

[5] T. Bartsch and D. G. de Figueiredo, "Infinitely many solutions of nonlinear elliptic systems," in Topics in Nonlinear Analysis, vol. 35 of Progress in Nonlinear Differential Equations and Their Applications, pp. 51-67, Birkhäuser, Basel, Switzerland, 1999.

[6] D. G. De Figueiredo and Y. H. Ding, "Strongly indefinite functionals and multiple solutions of elliptic systems," Transactions of the American Mathematical Society, vol. 355, pp. 2973-2989, 2003.

[7] D. G. Figueiredo, "Semilinear elliptic systems: existence, multiplicity, symmetry of solutions," in Handbook of Differential Equations: Stationary Partial Differential Equations, M. Chipot, Ed., vol. 5, pp. 1-47, Elsevier, 2008.

[8] S. I. Pohozaev and A. Tesei, "Existence of positive solutions to some nonlinear Neumann problems," Doklady Akademii Nauk SSSR, vol. 363, no. 4, pp. 450-453, 1998.

[9] S. I. Pohozaev and L. Véron, "Multiple positive solutions of some quasilinear Neumann problems," Applicable Analysis, vol. 74, no. 3-4, pp. 363-391, 2000.

[10] L. Toscano and S. Toscano, "On the solvability of a class of general systems of variational equations with nonmonotone operators," Journal of Interdisciplinary Mathematics, vol. 14, no. 2, pp. 123-147, 2011.

[11] L. Toscano and S. Toscano, "Dirichlet and neumann problems related to nonlinear elliptic systems: solvability, multiple solutions, solutions with positive components," Abstract and Applied Analysis, vol. 2012, Article ID 760854, 44 pages, 2012.

[12] S. I. Pohozaev, "On the global fibering method in nonlinear variational problems," Proceedings of the Steklov Institute of Mathematics, vol. 219, pp. 281-328, 1997.

[13] A. M. Piccirillo and R. Toscano, "Multiple solutions of some nonlinear elliptic problems containing the p-Laplacian," Differential Equations, vol. 37, no. 8, pp. 1121-1132, 2001.

[14] A. M. Piccirillo, L. Toscano, and S. Toscano, "On the solvability of some nonlinear Neumann problems," Advanced Nonlinear Studies, vol. 1, no. 1, pp. 102-120, 2001.

[15] A. M. Piccirillo, L. Toscano, and S. Toscano, "On the solvability of some nonlinear systems of elliptic equations," Advanced Nonlinear Studies, vol. 1, no. 2, pp. 89-115, 2001.

[16] A. Anane, "Simplicité et isolation de la première valeur propre du p-laplacian avec poids," Comptes Rendus de l'Académie des Sciences Series I: Mathematics, vol. 305, pp. 725-728, 1987.

[17] P. Tolksdorf, "Regularity for a more general class of quasilinear elliptic equations," Journal of Differential Equations, vol. 51, no. 1, pp. 126-150, 1984. 
[18] N. S. Trudinger, "On harnack type inequalities and their application to quasilinear elliptic equations," Communications on Pure and Applied Mathematics, vol. 20, no. 4, pp. 721-747, 1967. 


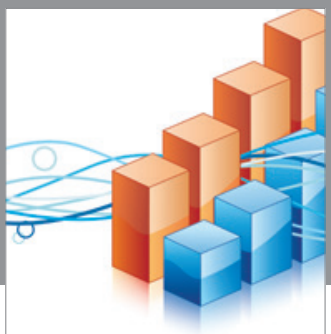

Advances in

Operations Research

vatem alat4

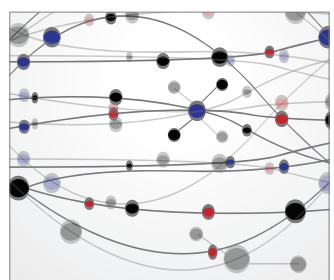

\section{The Scientific} World Journal
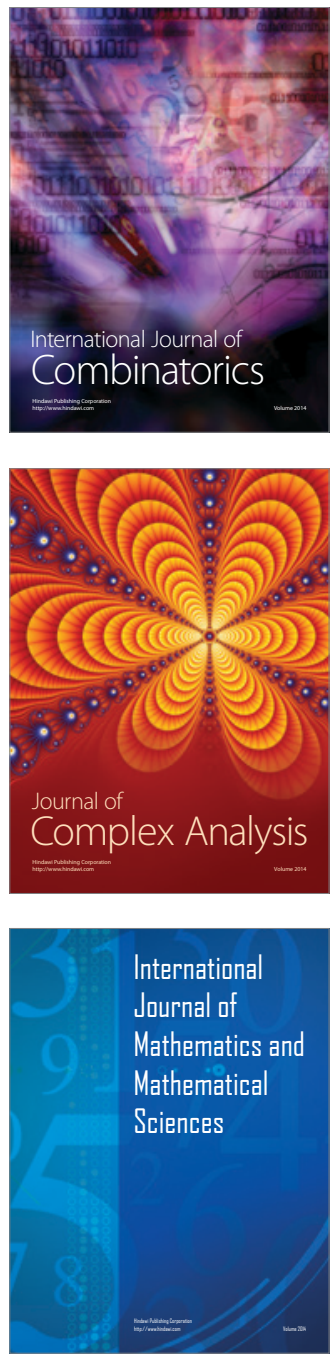
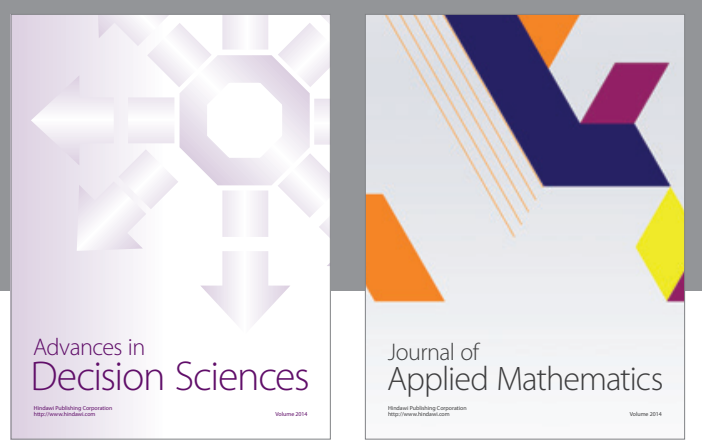

Algebra

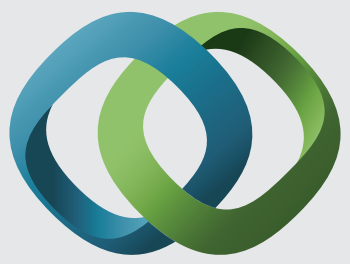

\section{Hindawi}

Submit your manuscripts at

http://www.hindawi.com
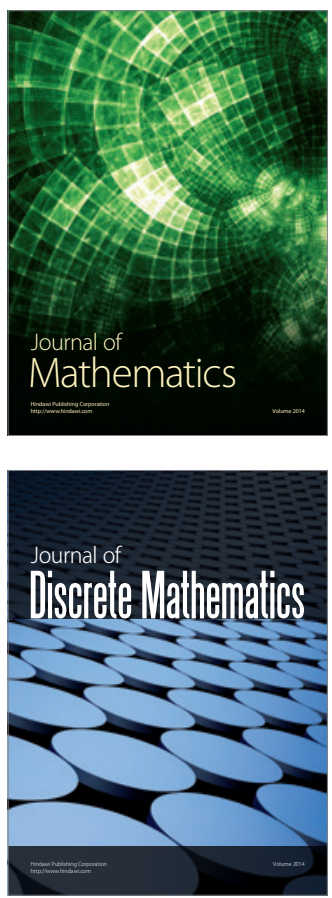

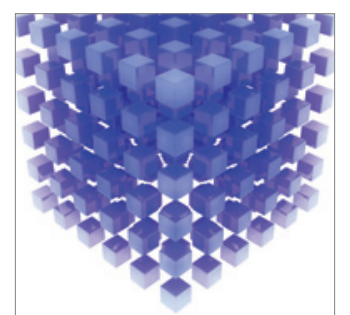

Mathematical Problems in Engineering
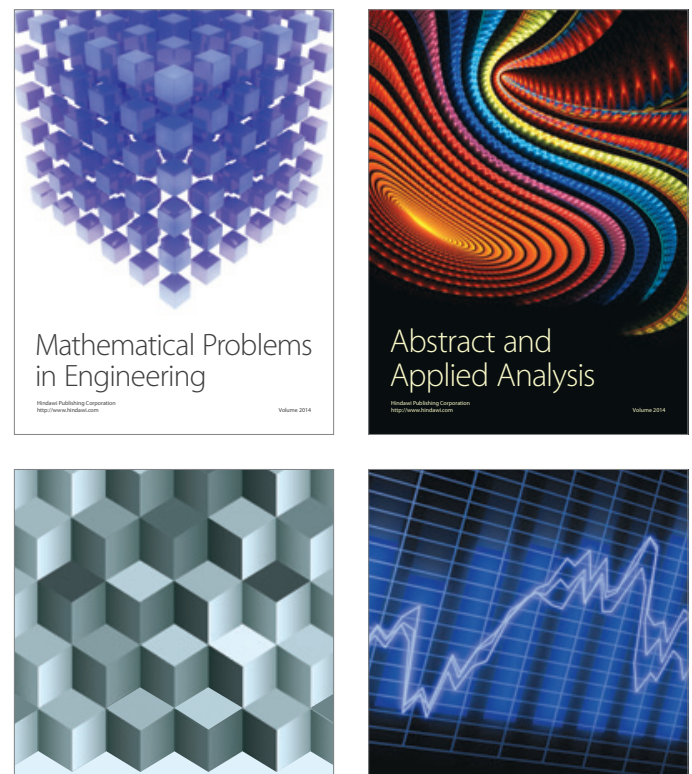

Journal of

Function Spaces

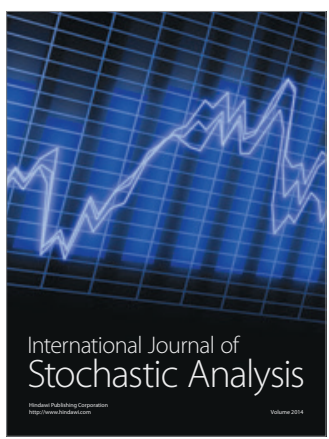

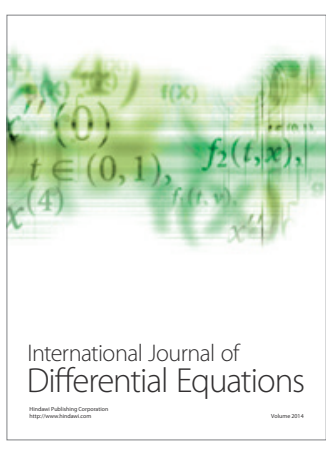
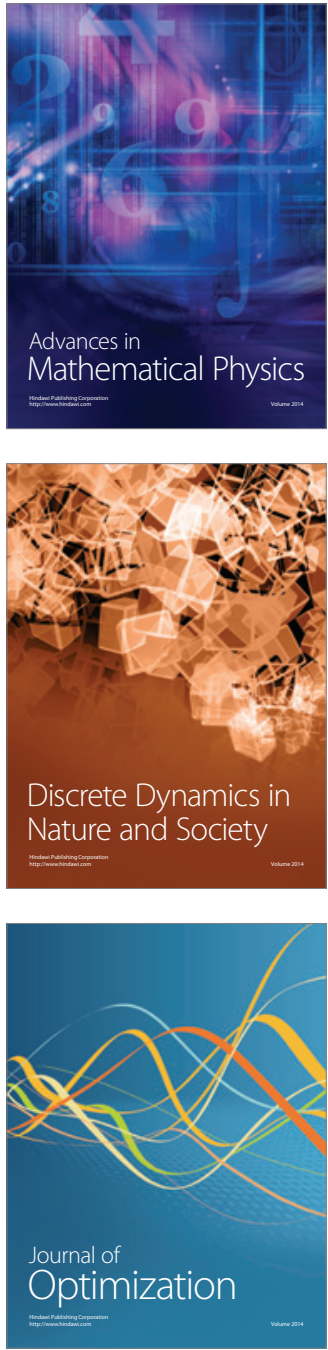